\title{
Thermalization with chemical potentials, and higher spin black holes
}

\author{
Gautam Mandal, Ritam Sinha, and Nilakash Sorokhaibam \\ Department of Theoretical Physics, \\ Tata Institute of Fundamental Research, Mumbai 400005, India \\ E-mail: mandal@theory.tifr.res.in, ritam@theory.tifr.res.in, \\ nilakashs@theory.tifr.res.in
}

ABSTRACT: We study the long time behaviour of local observables following a quantum quench in $1+1$ dimensional conformal field theories possessing additional conserved charges besides the energy. We show that the expectation value of an arbitrary string of local observables supported on a finite interval exponentially approaches an equilibrium value. The equilibrium is characterized by a temperature and chemical potentials defined in terms of the quenched state. For an infinite number of commuting conserved charges, the equilibrium ensemble is a generalized Gibbs ensemble (GGE). We compute the thermalization rate in a systematic perturbation in the chemical potentials, using a new technique to sum over an infinite number of Feynman diagrams. The above technique also allows us to compute relaxation times for thermal Green's functions in the presence of an arbitrary number of chemical potentials. In the context of a higher spin (hs $[\lambda]$ ) holography, the partition function of the final equilibrium GGE is known to agree with that of a higher spin black hole. The thermalization rate from the CFT computed in our paper agrees with the quasinormal frequency of a scalar field in this black hole.

Keywords: Field Theories in Lower Dimensions, AdS-CFT Correspondence, Boundary Quantum Field Theory, Conformal Field Models in String Theory

ARXIV EPRINT: 1501.04580 


\section{Contents}

1 Introduction and summary 1

2 One-point functions $\quad 6$

2.1 One-point function on the cylinder with chemical potentials 8

$\begin{array}{lll}2.2 & \text { One-point function on the strip with chemical potentials } & 10\end{array}$

$\begin{array}{lll}2.2 .1 & \mathcal{O}\left(\mu_{n}\right) \text { calculation } & 10\end{array}$

$\begin{array}{lll}2.2 .2 & \text { Higher order } \mu \text {-corrections } & 12\end{array}$

3 Calculation of $I(t) \quad \mathbf{1 5}$

$\begin{array}{ll}3.1 \text { Proof of thermalization } & 16\end{array}$

$\begin{array}{lll}3.2 & \text { Thermalization rate } & 17\end{array}$

$\begin{array}{ll}3.2 .1 \boldsymbol{\mu}=\mathbf{0} & 17\end{array}$

$\begin{array}{ll}3.2 .2 \mu \neq 0 & 18\end{array}$

$\begin{array}{lll}3.3 & \text { Properties of } \hat{Q} & 18\end{array}$

$\begin{array}{llr}4 & \text { Decay of perturbations of a thermal state } & 18\end{array}$

5 Holography and higher spin black holes 20

$\begin{array}{llr}6 & \text { Discussion } & 21\end{array}$

A Some details on one-point functions $\quad \mathbf{2 3}$

A.1 A few explicit one-point functions with zero chemical potentials 23

A.2 Some details on $O\left(\mu_{n}\right)$ correction to the one-point function 24

B Short interval expansion $\quad 26$

B.1 The coefficients $C_{k_{1}, k_{2}} \quad 27$

\section{Introduction and summary}

The study of thermalization in closed interacting quantum systems has a long history (see, e.g. [1] for a review). It has been known ever since the celebrated work of Fermi, Pasta and Ulam (FPU) that interacting classical systems need not necessarily equilibrate. The question of finding sufficient conditions for thermalization in quantum systems is also an open one. Recently, the advent of holography has linked the issue of thermalization in strongly coupled quantum field theories to another important, classical, problem of black hole formation (see, e.g. [2-5] and references therein). In the latter setting too, the issue of gravitational collapse of a given matter distribution is rather nontrivial; indeed there 
is an interesting debate in the current literature (see, e.g., [6-11]) regarding the fate of perturbations in anti-de-Sitter spacetimes.

In this paper, we will focus on two-dimensional conformal field theories (CFTs) on an infinite line $\sigma \in \mathbb{R}$. We will consider the system at $t=0$ to be in a "quenched state" 1

$$
\left|\psi_{0}\right\rangle=\exp \left[-\epsilon_{2} H-\sum_{n=3}^{\infty} \epsilon_{n} W_{n}\right]|B d\rangle
$$

Here $|B d\rangle$ is a conformal boundary state; the exponential factors cut off the UV modes to make the state normalizable. $W_{n}$ denote the additional conserved charges in the theory. ${ }^{2}$ This choice of the quenched state is a generalization of that in [12] for which $\epsilon_{n}=0$, for $n>3$.

The wavefunction for $t>0$ is given by

$$
|\psi(t)\rangle=\exp [-i H t]\left|\psi_{0}\right\rangle
$$

The questions we will explore, and answer, are: what is the long time behaviour of various observables in $|\psi(t)\rangle$ ? In particular, does the expectation value of an operator (or a string of operators) approach a constant? If so, (i) is the constant value characterized by a thermodynamic equilibrium, and (ii) what is the rate of approach to the constant value? More generally, we would also address, and partially answer, the questions: how does the existence and rate of thermalization depend on the initial state and the choice of observables?

Thermalization We find in this paper that the expectation values of local observables (supported on a finite interval $A: \sigma \in[-l / 2, l / 2]$ ) asymptotically approach (see (1.12) for the precise statement) their values in an equilibrium ensemble,

$$
\rho_{\text {eqm }}=\frac{1}{Z} \exp \left[-\beta H-\sum_{n} \mu_{n} W_{n}\right], \quad Z=\operatorname{Tr} \exp \left[-\beta H-\sum_{n} \mu_{n} W_{n}\right]
$$

whose temperature and chemical potentials are related to the cutoff scales in (1.5) as follows

$$
\beta=4 \epsilon_{2}, \quad \mu_{n}=4 \epsilon_{n}, \quad n=3,4, \ldots
$$

\footnotetext{
${ }^{1}$ In the original sense of the term, a quantum quench is defined as a sudden change from a hamiltonian $H_{0}$ to a hamiltonian $H$ which governs further evolution for $t \geq 0$. The system is assumed to be in the ground state of $H_{0}$ at $t=0$, which serves as an initial state for subsequent dynamics; the dynamics is nontrivial since the initial state prepared this way is not an eigenstate of $H$. In this paper, the state (1.1) is an ansatz for a quenched state, generalizing the Calabrese-Cardy (CC) ansatz [12]. The CC state has a single cut-off parameter $\epsilon_{2}$; this works for sudden quenches characterized by a single scale — the initial mass gap — which is much larger than the mass scale set by the cut-off parameter (see [13, 14] for subtleties associated with the 'sudden limit'). The generalized Calabrese-Cardy ansatz (1.1), accommodates quench protocols with multiple scales (see section 6). The other characteristic of the ansatz (1.1) is that it describes a global quench or a homogeneous quench, as the state is translationally invariant. We will briefly mention inhomogeneous and local quenches in section 6 .

${ }^{2}$ For the purposes of this paper, we will identify them with $W_{n}$-charges of $2 \mathrm{D}$ CFT, $n=3,4, \ldots$ (with $\left.W_{2} \equiv H\right)$, although much of what we say will go through independent of this specific choice as long as these charges mutually commute and are defined from currents which are quasiprimary fields of the conformal algebra.
} 
The relations (1.4) are uniquely dictated by the requirement that the expectation values of the conserved charges $H, W_{3}, W_{4}, \ldots$ in the initial state match those in the mixed state (1.3) (see (3.3)). In the absence of the extra parameters $\epsilon_{n}, n=3,4, \ldots$ this result is derived by the elegant method of conformal transformations [12]. In the presence of these parameters, this method is not available; in this paper, we deal with the extra exponential factors in terms of an infinite series and do a resummation.

We emphasize that the thermalization we found above persists even when we have an integrable model with an infinite number of conserved charges. Relaxation of local observables in integrable systems has been a very active subject of investigation in recent years; see, e.g. [15-25]. Specific models investigated in this context include (a) one-dimensional hardcore bosons [17], (b) transverse field Ising model [21], (c) Luttinger model [19], (d) matrix quantum mechanics [24], (e) sine-Gordon model [25], etc. The equilibrium ensembles in theses cases have been called a generalized Gibbs ensemble (GGE). Most of the above works involve quenches with a mass gap; however, ref. [19], and, closer to our context, ref. [23] (section 2.5), discuss possible extension to critical quenches involving post-quench conformal dynamics. ${ }^{3}$ The role of our present results is to quantitatively substantiate the occurrence of local thermalization in integrable conformal field theories for the general class of quenches described by (1.1), and to determine the relevant relaxation time scales, besides finding a holographic interpretation where applicable. Interestingly, the thermalization we find works even for free conformal field theories, e.g. a free scalar field theory. ${ }^{4}$

With the above identification of parameters, we will rewrite the initial quenched state (1.1) henceforth as

$$
\left|\psi_{0}\right\rangle=\exp \left[-\left(\beta H-\sum_{n=3}^{\infty} \mu_{n} W_{n}\right) / 4\right]|B d\rangle
$$

We find the following specific results.

1. Thermalization time scale for single local observables: we find that at large times

$$
\left\langle\psi(t)\left|\phi_{k}(\sigma)\right| \psi(t)\right\rangle=\operatorname{Tr}\left(\phi_{k}(0) \rho_{\mathrm{eqm}}\left(\beta, \mu_{i}\right)\right)+a_{k} e^{-\gamma_{k} t}+\ldots
$$

where $\phi_{k}(\sigma)$ is an arbitrary quasiprimary field (labelled by an index $k$ ). Below we compute the thermalization exponent $\gamma_{k}$ in a perturbation in the chemical potentials and to linear order it is given by

$$
\gamma_{k}=\frac{2 \pi}{\beta}\left[\Delta_{k}+\sum_{n} \tilde{\mu}_{n} Q_{n, k}+O\left(\tilde{\mu}^{2}\right)\right], \quad \tilde{\mu}_{n} \equiv \frac{\mu_{n}}{\beta^{n-1}},
$$

\footnotetext{
${ }^{3}$ It is of relevance here to refer to an important recent talk by John Cardy (see http://www.ggi.fi.infn.it/talkfiles/slides/talk2326.pdf) to which our attention was drawn by the anonymous referee after the arXiv version v2 of our paper was submitted for publication. This talk mentions unpublished results on thermalization involving deformation of Calabrese-Cardy states [12] by descendents and powers of the stress tensor. The issue addressed in this talk is similar in spirit to the broader question addressed in our sections 2 and 4, which includes integrable models with extended algebras.

${ }^{4}$ This happens essentially due to the fact that we consider here thermalization of local observables and that local field modes are mutually coupled even in a free field theory. Thermalization happens at times greater than the scale of localization, as we will see below.
} 
Here $\Delta_{k}=h_{k}+\bar{h}_{k}$ is the scaling dimension and $Q_{n, k}$ are the (shifted) $W_{n}$-charges (see (2.23) for the full definition) of the field $\phi_{k}$ (in case of primary fields) or of the minimum-dimension field which appears in the conformal transformation of $\phi_{k}$. To obtain this result, we perform the infinite resummation mentioned below (1.4). At large times, the perturbation series for the one-point function in the chemical potentials exponentiates (see (2.23)), to give the corrected exponent in the above equation. In various related contexts, finite orders of perturbation terms in chemical potentials have been computed before [26-28]. Our finding in this paper is that at large times, there is a regularity among the various orders leading to an exponential function as in (1.6) (see section 2.2.2 for details).

Universality: in the case of zero chemical potentials, it has been noted in [29], that although the relaxation time $\tau_{k}=2 \epsilon_{2} /\left(\pi \Delta_{k}\right)=\beta /\left(2 \pi \Delta_{k}\right)$ is non-universal (in the sense that it depends on the specific initial state (1.1)), the ratio of relaxation times for two different fields, namely, $\tau_{k_{1}} / \tau_{k_{2}}=\Delta_{k_{2}} / \Delta_{k_{1}}$ is universal (it depends only on the CFT data and not on the initial state and is hence expected to be valid for a general class of initial states). In the presence of the additional cut-off parameters $\epsilon_{i}, i=3, \ldots$ in the initial state (1.1), the ratio $\tau_{k_{1}} / \tau_{k_{2}}=\gamma_{k_{2}} / \gamma_{k_{1}}=\left(\Delta_{k_{2}}+\sum_{n} \tilde{\mu}_{n} Q_{n, k_{2}}\right) /\left(\Delta_{k_{1}}+\sum_{n} \tilde{\mu}_{n} Q_{n, k_{1}}\right)$ is, however, not independent of the initial state.

However, as we will briefly discuss in section 6, for a large class of quench states (e.g. where the energy density is uniform outside of a domain of compact support) the $\beta$-dependence of $\tau_{k}$, in the absence of chemical potentials, can be understood as the dependence on the uniform energy density (see a related discussion in [30]). The time scales $\tau_{k}$, therefore, do have a limited form of universality in the sense that it depends on a rather robust feature of the initial state. Our calculations in this paper leads us to believe that this feature will continue in the presence of chemical potentials, in the sense that the additional dependence of the time scales $1 / \gamma_{k}$ on the $\mu_{n}$ is fixed by the charge densities corresponding to the additional conserved charges. We hope to address this in [31].

2. Multiple local observables, reduced density matrix: besides the one-point functions discussed above, it turns our that we can demonstrate thermalization of all operators in an interval $A$ of length $l$. It is convenient to define a 'thermalization function' $I_{A}(t)[32]$ as

$$
\begin{aligned}
I_{A}(t) & =\operatorname{Tr}\left(\hat{\rho}_{\text {dyn }, A}(t) \hat{\rho}_{\text {eqm }, A}\left(\beta, \mu_{n}\right)\right)=\frac{\operatorname{Tr}\left(\rho_{\text {dyn }, A}(t) \rho_{\text {eqm }, A}\left(\beta, \mu_{n}\right)\right)}{\left[\operatorname{Tr}\left(\rho_{\text {dyn }, A}(t)^{2}\right) \operatorname{Tr}\left(\rho_{\text {eqm }, A}\left(\beta, \mu_{i}\right)^{2}\right)\right]^{1 / 2}} \\
\rho_{\text {dyn }, A}(t) & =\operatorname{Tr}_{\bar{A}}|\psi(t)\rangle\langle\psi(t)|, \quad \rho_{\text {eqm }, A}\left(\beta, \mu_{n}\right)=\operatorname{Tr}_{\bar{A}} \rho_{\text {eqm }}\left(\beta, \mu_{i}\right)
\end{aligned}
$$

Here $\hat{\rho}=\rho / \sqrt{\operatorname{Tr} \rho^{2}}$ denotes a 'square-normalized' density matrix. ${ }^{56}$ We show below that at large times the thermalization function has the form

$$
I_{A}(t)=1-\alpha(\tilde{l}) e^{-2 \gamma_{m} t}+\ldots, \tilde{l} \equiv l / \beta
$$

\footnotetext{
${ }^{5}$ Note that operators in a Hilbert space $\mathrm{H}$ can themselves be regarded as vectors in $\mathbf{H} \times \mathrm{H}^{*}$; under this interpretation $\operatorname{Tr}(A B)$ defines a positive definite scalar product. With this understanding, we will regard the hatted density matrices as unit vectors.

${ }^{6}$ Throughout this paper, we will consider field theories with an infinite spatial extent. The entire Hilbert space is assumed to be of the form $\mathrm{H}_{A} \otimes \mathrm{H}_{\bar{A}}$. $\operatorname{Tr}_{\bar{A}}$ implies tracing over $\mathrm{H}_{\bar{A}}$.
} 
where $\gamma_{m}$ refers to the exponent (1.7) for the operator $\phi_{m}$ with minimum scaling dimension. $^{7} \alpha(\tilde{l})$ is computed as a power series in $\tilde{l}$ which we find using the short interval expansion, valid for $\tilde{l} \ll 1$, i.e. $l \ll \beta$.

Two immediate consequences of (1.9) are

(i) Thermalization of an arbitrary string of operators: note, from (1.9), that

$$
I_{A}(t) \stackrel{t \rightarrow \infty}{\longrightarrow} 1,
$$

Since the square-normalized density matrices can be regarded as unit vectors (in the sense of footnote 5 ), and $I_{A}(t)$ can be regarded as the scalar product $\hat{\rho}_{\mathrm{dyn}, A}(t) \cdot \hat{\rho}_{\mathrm{eqm}, A},(1.10)$ clearly implies

$$
\hat{\rho}_{\mathrm{dyn}, A}(t) \stackrel{t \rightarrow \infty}{\longrightarrow} \hat{\rho}_{\mathrm{eqm}, A}
$$

This implies the following statement of thermalization for an arbitrary string of local operators (with $\sigma_{1}, \sigma_{2}, \ldots \in A$ )

$$
\begin{aligned}
\left\langle\psi(t)\left|O\left(\sigma_{1}, t_{1}\right) O\left(\sigma_{2}, t_{2}\right) \ldots\right| \psi(t)\right\rangle=\operatorname{Tr}\left(\hat{\rho}_{\mathrm{dyn}, A}(t) O\left(\sigma_{1}, t_{1}\right) O\left(\sigma_{2}, t_{2}\right) \ldots\right) & \\
\stackrel{t \rightarrow \infty}{\longrightarrow} & \operatorname{Tr}\left(\hat{\rho}_{\mathrm{eqm}, A} O\left(\sigma_{1}, t_{1}\right) O\left(\sigma_{2}, t_{2}\right) \ldots\right) .
\end{aligned}
$$

(ii) Long time behaviour of reduced density matrix: carrying on with the interpretation of $I_{A}(t)$ as a scalar product, we can infer following asymptotic behaviour of $\hat{\rho}_{\text {dyn }}(t)$ from (1.9):

$$
\hat{\rho}_{\mathrm{dyn}, A}(t)=\hat{\rho}_{\mathrm{eqm}, A}\left(\beta, \mu_{i}\right)\left(1-\alpha e^{-2 \gamma_{m} t}+\ldots\right)+\hat{Q}\left(\sqrt{2 \alpha} e^{-\gamma_{m} t}+\ldots\right)
$$

where $\operatorname{Tr}\left(\hat{Q}^{2}\right)=1, \quad \operatorname{Tr}\left(\hat{\rho}_{\text {eqm }, A}\left(\beta, \mu_{i}\right) \hat{Q}\right)=0$. We will specify further properties of $\hat{Q}$ later on.

Importance of local observables: in case of a free massless scalar field, it is easy to show that quantities like $\left\langle\psi(t)\left|\alpha_{1}^{2} \alpha_{1}^{\dagger}\right| \psi(t)\right\rangle$ perpetually oscillate and never reach a constant (see a related calculation in [24]). The modes $\alpha_{n}$ represent Fourier modes and are non-local. Indeed, as $[29,32,33]$ showed, in the absence of chemical potentials, the exponential term in $(1.9)$ is $e^{-2 \gamma_{m}(t-l / 2)}$ and the thermalization sets in only after $t$ exceeds $l / 2$. Thus, for $l=\infty$, there is no thermalization, which is consistent with the above observation about perpetual oscillations. We expect the form $e^{-2 \gamma_{m}(t-l / 2)}$ to continue to hold in the presence of chemical potentials ${ }^{8}$, since the effect of the chemical potentials on the exponent $\gamma_{k}$ can be viewed as a shift of the

\footnotetext{
${ }^{7}$ We will assume here that the spectrum of such $\Delta$ 's is bounded below by a finite positive number. In case of a free scalar field theory, we can achieve this by considering a compactified target space.

${ }^{8}$ Although, in the short-interval expansion employed in this paper to derive (1.9), which uses $t \gg \beta \gg l$, such an $l$-dependence in the exponent cannot be easily seen from the pre-factor $\alpha(\tilde{l})$ unless one sums over an infinite orders in $\tilde{l}$.
} 
anomalous dimension $\delta \Delta_{k}=\sum_{n} \tilde{\mu}_{n} Q_{n, k}+O\left(\tilde{\mu}^{2}\right)$ (see, e.g. (4.8)). This shows that, as in the case of zero chemical potentials, equilibration sets in only after $t$ exceeds $l / 2$. We will see a similar phenomena next in the context of a decay of perturbations to a thermal state.

3. Decay of perturbations to a thermal state: we compute (see section 4 for details) the time-dependent two-point Green's function $G_{+}(t, l ; \beta, \mu)$ for two points spatially separated by a distance $l$. We find that for $t, l, t-l \gg \beta$, the time-dependence is exponential, with the same exponent as in (1.6):

$$
G_{+}(t, l ; \beta, \mu) \equiv \frac{1}{Z} \operatorname{Tr}\left(\phi_{k}(l, t) \phi_{k}(0,0) e^{-\beta H-\sum_{n} \mu_{n} W_{n}}\right)=\text { const } e^{-\gamma_{k} t}
$$

Note that the above thermalization sets in for $t>l$. For $t<l$, the two-point function has an exponential decay in the spatial separation (see section 4 and figure 3 ).

The computation of the above relaxation times in the presence of an arbitrary number of chemical potentials uses the technique, described above, of summing over an infinite number of Feynman diagrams, and is one of the main results of our paper.

4. Collapse to higher spin black holes: in $[34,35]$ the bulk dual to the time-dependent state (1.2) corresponding to initial condition (1.5), for large central charges, has been constructed in the case of zero chemical potentials. The dual geometry corresponds to one half of the eternal BTZ (black string) geometry, whose boundary represents an end-of-theworld brane. In [36] the result has been extended to the case of non-zero angular momentum and a Chern-Simons charge. In case of an infinite number of chemical potentials, a bulk dual to the equilibrium ensemble (1.3) has been identified, in the context of the GaberdielGopakumar hs $(\lambda)$ theory [37], as a higher spin black hole with those chemical potentials [38, $39]$. It is natural to conjecture $[24,36]$ that the time-development $(1.2)$ should be dual to a collapse to this higher spin black hole. At late times, therefore, the thermalization exponent found above should correspond to the quasinormal frequency of the higher spin black hole. We find that (see section 5 and [40]) this is indeed borne out in a specific example.

The plan of the paper is as follows. The results 1,2,3 and 4 above are described in sections 2, 3, 4 and 5, respectively. The resummation of an infinite number of Feynman diagrams (corresponding to insertions of arbitrary number of chemical potential terms) is discussed in section 2.2.2, which uses results in appendix A. The calculation of the overlap of reduced density matrices in section 3 needs the use of the short-interval expansion, which is described in appendix B. In section 6 we present our conclusions and make some remarks on inhomogeneous quench [31].

\section{One-point functions}

In this section we will consider the behaviour of the following one-point functions of a quasiprimary field $\phi_{k}(\sigma)$

$$
\begin{aligned}
\left\langle\phi_{k}(\sigma, t)\right\rangle_{\mathrm{dyn}} & \equiv\left\langle\psi(t)\left|\phi_{k}(\sigma)\right| \psi(t)\right\rangle, \\
\left\langle\phi_{k}(\sigma)\right\rangle_{\mathrm{eqm}} & \equiv \operatorname{Tr}\left(\phi_{k}(\sigma) \rho_{\mathrm{eqm}}\left(\beta, \mu_{n}\right)\right)
\end{aligned}
$$


We will briefly recall how these are computed in the absence of the chemical potentials $[12$, 41]. The first expectation value corresponds to the one-point function on a strip geometry, with complex coordinate $w=\sigma+i \tau, \sigma \in(-\infty, \infty), \tau \in(-\beta / 4, \beta / 4)$ where $\tau$ is eventually to be analytically continued to $\tau=i t$. This can be conformally transformed to an upper half plane by using the map

$$
z=i e^{(2 \pi / \beta) w}
$$

For a primary field with $h_{k}=\bar{h}_{k}$ (of the form $\phi_{k}(w, \bar{w})=\varphi_{k}(w) \varphi_{k}(\bar{w})$ ), this procedure gives $^{9}$ (for other primary fields, the one-point function vanishes)

$$
\begin{aligned}
\left\langle\phi_{k}(\sigma, t)\right\rangle_{\mathrm{dyn}} & =\left\langle\phi_{k}(w, \bar{w})\right\rangle_{\mathrm{str}}=\left(\frac{\partial z}{\partial w}\right)^{h_{k}}\left(\frac{\partial \bar{z}}{\partial \bar{w}}\right)^{\bar{h}_{k}}\left\langle\phi_{k}(z, \bar{z})\right\rangle_{U H P} \\
& =a_{k}\left(e^{2 \pi t / \beta}+e^{-2 \pi t / \beta}\right)^{-2 h_{k}} \sim a_{k} e^{-\gamma_{k}^{(0)} t}+\ldots, \gamma_{k}^{(0)}=2 \pi \Delta_{k} / \beta=4 \pi h_{k} / \beta
\end{aligned}
$$

We have used the following result for the one-point function on the UHP:

$$
\left\langle\phi_{k}(z, \bar{z})\right\rangle_{U H P}=A_{k}\left\langle\varphi_{k}(z) \varphi_{k}^{*}\left(z^{\prime}\right)\right\rangle_{U H P}=A_{k}\left(z-z^{\prime}\right)^{-2 h_{k}}, h_{k}=\bar{h}_{k}, z^{\prime}=\bar{z}
$$

which follows by using the method of images where the antiholomorphic factor of $\phi_{k}(z, \bar{z})$ on the upper half plane at the point $(z, \bar{z})$ is mapped (up to a constant) to the holomorphic $\varphi_{k}^{* 10}$ on the lower half plane at the image point $\left(z^{\prime}, \bar{z}^{\prime}\right)$ with $z^{\prime}=\bar{z}, \bar{z}^{\prime}=z[41,42]$. In the above $a_{k}, A_{k}$ are known numerical constants. Note that

$$
z=i e^{2 \pi(\sigma+i \tau) / \beta}=i e^{2 \pi(\sigma-t) / \beta}, \quad z^{\prime}=\bar{z}=-i e^{2 \pi(\sigma-i \tau) / \beta}=-i e^{2 \pi(\sigma+t) / \beta}
$$

so that in the large time limit we have

$$
t \rightarrow \infty \Rightarrow z \rightarrow 0, \bar{z} \rightarrow-i \infty .
$$

The second, thermal, expectation value in (2.1), for $\mu_{n}=0$, corresponds to a cylindrical geometry in the $w$-plane, with $\tau=0$ identified with $\tau=\beta$. By using the same conformal map (2.2) this can be transformed to a one-point function on the plane. For a primary field the latter vanishes. Hence (1.6) is trivially satisfied.

For a quasiprimary field $\phi_{k}$, its conformal transformation generates additional terms, including possibly a c-number term $c_{k}$ (e.g. the Schwarzian derivative term for $\phi_{k}=T_{w w}$ ) and generically lower order operators. The c-number term does not distinguish between a plane and an UHP. This leads to the following overall result (for $\mu_{n}=0$ ):

$$
\begin{aligned}
\left\langle\phi_{k}(\sigma)\right\rangle_{\mathrm{eqm}} & =\left\langle\phi_{k}(w, \bar{w})\right\rangle_{\mathrm{cyl}}=c_{k}, \\
\left\langle\phi_{k}(\sigma, t)\right\rangle_{\mathrm{dyn}} & =\left\langle\phi_{k}(w, \bar{w})\right\rangle_{\mathrm{str}}=c_{k}+a_{k} e^{-\gamma_{k}^{(0)} t}+\ldots, \quad \gamma_{k}^{(0)}=2 \pi \Delta_{k} / \beta,
\end{aligned}
$$

\footnotetext{
${ }^{9}$ The subscripts str, cyl will denote a 'strip' and a 'cylinder', respectively.

${ }^{10} \mathrm{We}$ distinguish $\varphi_{k}^{*}$ from $\varphi_{k}$ to allow for charge conjugation.
} 
where $\Delta_{k}$ now is the scaling dimension of the minimum-dimension operator in a $T\left(z_{1}\right) \phi_{k}(z)$ OPE. This is clearly of the general form (1.6) for $\mu_{n}=0$.

We now turn to a discussion of these expectation values (2.1) in the presence of chemical potentials $\mu_{n}, n=3,4, \ldots$, as in (1.5) and (1.3). We will denote the new conserved currents as $\mathcal{W}_{n}(w)$ and $\overline{\mathcal{W}}_{n}(\bar{w}), n=3,4, \ldots$. The conserved charge, $W_{n}$, is defined as

$$
W_{n}=\frac{1}{2 \pi} \int_{\Gamma} W_{\tau \tau \ldots \tau} d \sigma=\frac{1}{2 \pi} \int_{\Gamma}\left(i^{n} d w_{1} \mathcal{W}_{n}\left(w_{1}\right)+(-i)^{n} d \bar{w}_{1} \overline{\mathcal{W}}_{n}\left(\bar{w}_{1}\right)\right)
$$

Here the contour $\Gamma$ is taken to be a $\tau=$ constant line along which $d w_{1}=d \bar{w}_{1}=d \sigma$. Under the conformal transformation (2.2) to the plane/UHP, the holomorphic part of the contour integral becomes

$$
\left.W_{n}\right|_{h o l}=\frac{i^{n}}{2 \pi}\left(\frac{2 \pi}{\beta}\right)^{n-1} \int_{\Gamma_{1}} d z_{1}\left[z_{1}^{n-1} \mathcal{W}_{n}\left(z_{1}\right)+\sum_{m=1}^{\lfloor n / 2\rfloor} a_{n, n-2 m} z_{1}^{n-2 m-1} \mathcal{W}_{n-2 m}\left(z_{1}\right)\right]
$$

where the $a_{n, n-2 m}$ denote the mixing of $\mathcal{W}_{n}\left(z_{1}\right)$ with lower order $W$-currents under conformal transformations $[43,44]$. The contour $\Gamma_{1}$ is an image of the contour $\Gamma$ onto the plane. The expression for the antiholomorphic part $\left.W_{n}\right|_{\text {antihol }}$ is similar.

As mentioned before, in this paper we will regard the $W_{n}$ as conserved charges of a $\mathrm{W}$-algebra, although the results we derive will be equally valid as long as these charges, together with $H$, form a mutually commuting set, and the currents $\left(\mathcal{W}_{n}(w), \overline{\mathcal{W}}_{n}(\bar{w})\right)$ are quasiprimary fields.

\subsection{One-point function on the cylinder with chemical potentials}

For simplicity we first consider the equilibrium expectation value in (2.1). Unfortunately, unlike the thermal factor above, the factor $e^{-\sum_{n} \mu_{n} W_{n}}$ in (1.3) cannot be dealt with in terms of a conformal map. We will, therefore, treat this factor as an operator insertion, and write

$$
\left\langle\phi_{k}(\sigma)\right\rangle_{\mathrm{eqm}} \equiv \operatorname{Tr}\left(\phi_{k}(w, \bar{w}) \rho_{\mathrm{eqm}}\left(\beta, \mu_{n}\right)\right)=\frac{\left\langle e^{-\sum_{n} \mu_{n} W_{n}} \phi_{k}(w, \bar{w})\right\rangle_{\mathrm{cyl}}}{\left\langle e^{-\sum_{n} \mu_{n} W_{n}}\right\rangle_{\mathrm{cyl}}} \equiv\left\langle\phi_{k}(w, \bar{w})\right\rangle_{\mathrm{cyl}}^{\mu}
$$

We will now illustrate how to compute this for a single chemical potential, say $\mu_{3}$, using perturbation theory Feynman diagrams: ${ }^{11}$

$$
\left\langle\phi_{k}(w, \bar{w})\right\rangle_{\mathrm{cyl}}^{\mu}=\left\langle\phi_{k}(w, \bar{w})\right\rangle_{\mathrm{cyl}}-\mu_{3}\left\langle W_{3} \phi_{k}(w, \bar{w})\right\rangle_{\mathrm{cyl}}^{\mathrm{conn}}+\frac{\mu_{3}^{2}}{2 !}\left\langle W_{3} W_{3} \phi_{k}(w, \bar{w})\right\rangle_{\mathrm{cyl}}^{\mathrm{conn}}+\mathcal{O}\left(\mu_{3}^{3}\right)
$$

The first term in the above expression is the constant $c_{k}$ that we already encountered in (2.7). For a holomorphic primary field $\phi_{k}$, the second, $O\left(\mu_{3}\right)$, term, transformed on to the plane, gives

$$
\begin{aligned}
\left\langle W_{3} \phi_{k}(w)\right\rangle_{\mathrm{cyl}}^{\mathrm{conn}}=\frac{2 \pi}{\beta^{2}} z^{h_{k}}\left[i^{3} \int_{\Gamma_{1}}\right. & d z_{1} z_{1}^{2}\left\langle\mathcal{W}_{3}\left(z_{1}\right) \phi_{k}(z)\right\rangle_{\mathbb{C}}^{\mathrm{conn}} \\
& \left.+(-i)^{3} \int_{\Gamma_{1}} d \bar{z}_{1} \bar{z}_{1}^{2}\left\langle\overline{\mathcal{W}}_{3}\left(\bar{z}_{1}\right) \phi_{k}(z)\right\rangle_{\mathbb{C}}^{\text {conn }}\right]
\end{aligned}
$$

\footnotetext{
${ }^{11}$ The superscript conn denotes 'connected'.
} 
Here we have used the contour representations (2.8) and (2.9). The correlator inside the second integral obviously vanishes (it factorizes into a holomorphic and an antiholomorphic one-point functions, leading to a vanishing connected part). The first integral vanishes unless $\phi_{k}=\mathcal{W}_{3}$ (this uses the orthogonality of the basis of quasiprimary fields). In the latter case, using

$$
\left\langle\mathcal{W}_{3}\left(z_{1}\right) \mathcal{W}_{3}(z)\right\rangle_{\mathbb{C}}=\frac{c / 3}{\left(z_{1}-z\right)^{6}}
$$

the integral evaluates to $c /\left(90 z^{3}\right)$; combining with the factor of $z^{3}$ outside $\left(h_{k}=3\right.$ in this case) we get a $z$-independent constant, as we must, because of translational invariance on the plane. With an antiholomorphic primary field $\phi_{k}$, the calculation is isomorphic. For a primary field with nonvanishing $h_{k}, \bar{h}_{k}$ the result vanishes. For quasiprimary $\phi_{k}$, as well as for other $W_{n}$ charges, the conformal transformation to the plane additionally generates lower order operators (see, e.g. (2.9))), each of which can be dealt with as in (2.12). The result is a finite constant which we will denote as

$$
\left\langle W_{n} \phi_{k}(w, \bar{w})\right\rangle=c_{n, k}
$$

(this will be non-vanishing only for special choices of $\phi_{k}$, e.g. $\phi_{k}=\mathcal{W}_{n}$ ). As explained above, for $n=3$ and $\phi_{k}(w, \bar{w})=\mathcal{W}_{3}(w), c_{n, k}=-2 \pi c /\left(90 \beta^{2}\right)$.

In a similar fashion, the $O\left(\mu_{3}^{2}\right)$ term in (2.11) can be transformed to the plane. Again, we present the explicit expression for the simple case of a holomorphic primary field $\phi_{k}$.

$$
\begin{aligned}
\left\langle W_{3} W_{3} \phi_{k}(w)\right\rangle_{\mathrm{cyl}}^{\text {conn }}=( & \left.\frac{2 \pi}{\beta^{2}}\right)^{2} z^{h_{k}}\left[i^{6} \int_{\Gamma_{1}} d z_{1} \int_{\Gamma_{2}} d z_{2}\left\langle\mathcal{W}_{3}\left(z_{1}\right) \mathcal{W}_{3}\left(z_{2}\right) \phi_{k}(z)\right\rangle_{\mathbb{C}}^{\text {conn }} z_{1}^{2} z_{2}^{2}\right. \\
& +(-i)^{6} \int_{\Gamma_{1}} d \bar{z}_{1} \int_{\Gamma_{2}} d \bar{z}_{2}\left\langle\overline{\mathcal{W}}_{3}\left(\bar{z}_{1}\right) \overline{\mathcal{W}}_{3}\left(\bar{z}_{2}\right) \phi_{k}(z)\right\rangle_{\mathbb{C}}^{\mathrm{conn}} \bar{z}_{1}^{2} \bar{z}_{2}^{2} \\
& +\int_{\Gamma_{1}} d z_{1} \int_{\Gamma_{2}} d \bar{z}_{2}\left\langle\mathcal{W}_{3}\left(z_{1}\right) \overline{\mathcal{W}}_{3}\left(\bar{z}_{2}\right) \phi_{k}(z)\right\rangle_{\mathbb{C}}^{\mathrm{conn}} z_{1}^{2} \bar{z}_{2}^{2} \\
& \left.+\int_{\Gamma_{1}} d \bar{z}_{1} \int_{\Gamma_{2}} d z_{2}\left\langle\overline{\mathcal{W}}_{3}\left(\bar{z}_{1}\right) \mathcal{W}_{3}\left(z_{2}\right) \phi_{k}(z)\right\rangle_{\mathbb{C}}^{\text {conn }} \bar{z}_{1}^{2} z_{2}^{2}\right]
\end{aligned}
$$

For holomorphic quasiprimary $\phi_{k}$, additional, similar, terms appear due to the generation of lower order operators under conformal transformation to the plane. Only the holomorphic correlator survives (as in the $\mathrm{O}\left(\mu_{3}\right)$ calculation). Thus, e.g. if $\phi_{k}=T(z)$, the stress tensor, we have

$$
\left\langle\mathcal{W}_{3}\left(z_{1}\right) \mathcal{W}_{3}\left(z_{2}\right) T(z)\right\rangle_{\mathbb{C}}=\frac{c}{\left(z_{1}-z_{2}\right)^{4}\left(z_{1}-z\right)^{2}\left(z_{2}-z\right)^{2}}
$$

Again, after performing the integration over $z_{1}$ and $z_{2}$, we obtain a $z$-independent constant, as we must. The analysis of more general fields $\phi_{k}$ and two arbitrary $W$-charges is straightforwardly generalizable. The result is a finite constant (can be zero for a particular $\left.\phi_{k}\right)$ which we denote as

$$
\left\langle W_{m} W_{n} \phi_{k}(w, \bar{w})\right\rangle=c_{m n, k}
$$

Note that in (2.13) the result does not depend on the location of the contours $\Gamma_{1}, \Gamma_{2}$ on the plane, since the $W$-currents are conserved. 
Summarizing, we get

$$
\left\langle\phi_{k}(w, \bar{w})\right\rangle_{\mathrm{cyl}}^{\mu}=c_{k}-\sum_{n} \mu_{n} c_{n, k}+\frac{1}{2 !} \sum_{m, n} \mu_{m} \mu_{n} c_{m n, k}+O\left(\mu^{3}\right)
$$

\subsection{One-point function on the strip with chemical potentials}

Similarly to the previous subsection, we will treat the $\mu$-deformations in (1.5) as operator insertions:

$$
\begin{aligned}
\left\langle\phi_{k}(\sigma, t)\right\rangle_{\mathrm{dyn}} \equiv\left\langle\psi(t)\left|\phi_{k}(\sigma)\right| \psi(t)\right\rangle & =\frac{\left\langle e^{-\sum_{n} \mu_{n} W_{n} / 4} \phi_{k}(w, \bar{w}) e^{-\sum_{n} \mu_{n} W_{n} / 4}\right\rangle_{\mathrm{str}}}{\left\langle e^{-\sum_{n} \mu_{n} W_{n} / 2}\right\rangle_{\mathrm{str}}} \\
& \equiv\left\langle\phi_{k}(w, \bar{w})\right\rangle_{\mathrm{str}}^{\mu}
\end{aligned}
$$

As before, we begin by illustrating the calculation of this quantity with the simplest case of a single chemical potential $\mu_{3}$, using perturbation theory Feynman diagrams:

$$
\begin{aligned}
\left\langle\phi_{k}(w, \bar{w})\right\rangle_{\mathrm{str}}^{\mu}= & \left\langle\phi_{k}(w, \bar{w})\right\rangle_{\mathrm{str}}-\frac{\mu_{3}}{4}\left\langle\left\{W_{3}, \phi_{k}(w, \bar{w})\right\}\right\rangle_{\mathrm{str}}^{\mathrm{conn}} \\
& +\left(\frac{\mu_{3}}{4}\right)^{2} \frac{1}{2 !}\left(\left\langle\left\{W_{3} W_{3}, \phi_{k}(w, \bar{w})\right\}\right\rangle_{\mathrm{str}}^{\mathrm{conn}}+2\left\langle W_{3} \phi_{k}(w, \bar{w}) W_{3}\right\rangle_{\mathrm{str}}^{\mathrm{conn}}\right)+\mathcal{O}\left(\mu_{n}^{3}\right)
\end{aligned}
$$

The $\{$,$\} denotes an anticommutator. The operator ordering implies the following: when$ $W_{3}$ appears on the left of $\phi_{k}(w, \bar{w})$, e.g., in $\left\langle W_{3} \phi_{k}(w, \bar{w})\right\rangle$, the integration contour (2.8) for $W_{3}$ on the strip lies above the point $(w, \bar{w})$; similarly when $W_{3}$ appears on the right of $\phi_{k}(w, \bar{w})$, e.g. in $\left\langle\phi_{k}(w, \bar{w}) W_{3}\right\rangle$, the contour for $W_{3}$ is below the point $(w, \bar{w})$.

The first, $\mu$-independent, term in the above expansion is already calculated in (2.7).

\subsection{1 $\mathcal{O}\left(\mu_{n}\right)$ calculation}

As before, we find it convenient to use the conformal transformation (2.2). The correlator on the strip then reduces to that on the UHP, as in the $\mu=0$ case before. For a holomorphic primary field $\phi_{k}$, this gives

$$
\begin{aligned}
\left\langle W_{3} \phi_{k}(w)\right\rangle_{\mathrm{str}}^{\mathrm{conn}}=\frac{2 \pi}{\beta^{2}} z^{h_{k}}\left[i^{3} \int_{\Gamma_{1}} d z_{1} z_{1}^{2}\left\langle\mathcal{W} \mathcal{W}_{3}\left(z_{1}\right) \phi_{k}(z)\right\rangle_{\mathrm{UHP}}^{\mathrm{conn}}\right. \\
\\
\left.+(-i)^{3} \int_{\Gamma_{1}} d \bar{z}_{1} \bar{z}_{1}^{2}\left\langle\overline{\mathcal{W}}_{3}\left(\bar{z}_{1}\right) \phi_{k}(z)\right\rangle_{\mathrm{UHP}}^{\text {conn }}\right]
\end{aligned}
$$

where the operator ordering explained above implies that the contour $\Gamma_{1}$ lies to the left of the point $(z, \bar{z})$ on the UHP. Now, in the analogous calculation (2.12), the second connected correlator on the complex plane vanished because of factorization into one-point functions. Correlators on the UHP are, however, related to those on the plane by the method of images (an example of which we saw in (2.4)). In particular, $\overline{\mathcal{W}}_{3}$ at the point $\left(z_{1}, \bar{z}_{1}\right)$ on the UHP becomes the holomorphic operator $\mathcal{W}_{3}^{*}=-\mathcal{W}_{3}$ on the LHP at the point $\left(z_{1}^{\prime}, \bar{z}_{1}^{\prime}\right)$ with $z_{1}^{\prime}=\bar{z}_{1}[41,42]$. The contour $\Gamma_{1}$ gets mapped to its mirror image $\Gamma_{1}^{\prime}$ on the lower half plane. With this, we get

$$
\left\langle W_{3} \phi_{k}(w)\right\rangle_{\mathrm{str}}^{\mathrm{conn}}=\frac{2 \pi}{\beta^{2}} z^{h_{k}}\left[i^{3} \int_{\Gamma_{1}+\Gamma_{1}^{\prime}} d z_{1} z_{1}^{2}\left\langle\mathcal{W}_{3}\left(z_{1}\right) \phi_{k}(z)\right\rangle_{\mathbb{C}}^{\mathrm{conn}}\right]
$$




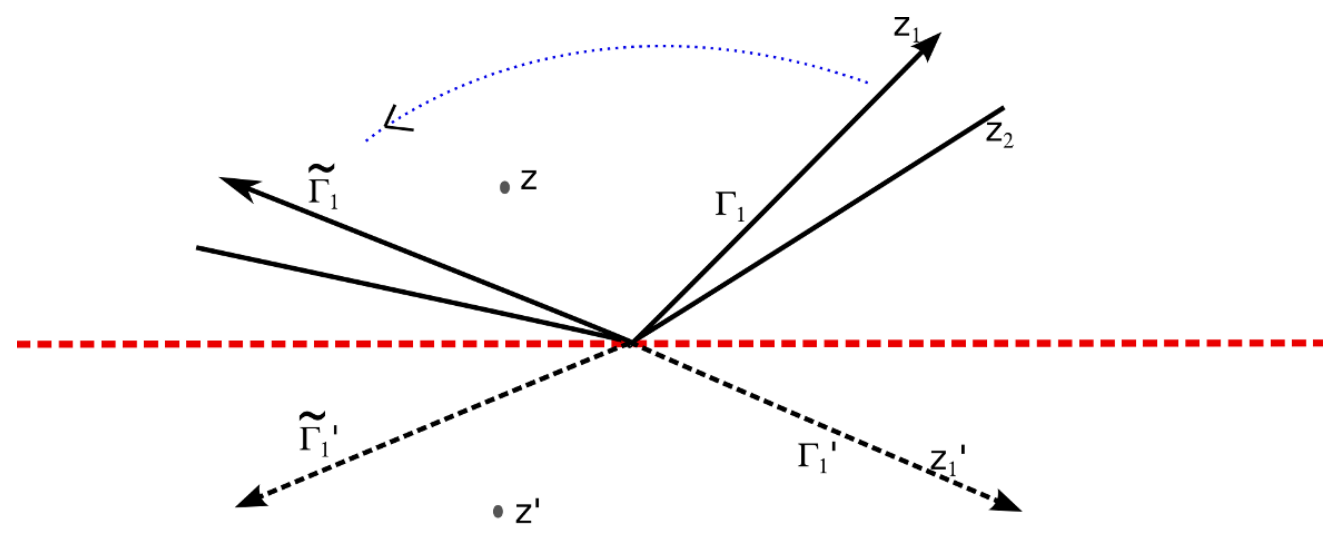

Figure 1. Various contours needed to compute the $W_{n}$ insertions in (2.16). At late times, the insertion of each contour, irrespective of the position of the contour, amounts to insertion of a given factor linear in $t$. This allows to re-sum arbitrary orders of arbitrary $W_{n}$-charge insertions, leading to the exponential time-dependence as in (1.6). See figure 2 for more.

On the complex plane, the contour $\Gamma_{1}$ on the UHP can be deformed to $\Gamma_{1}^{\prime}$ on the LHP, hence the two contours simply yield a factor of 2 . In fact, combining with the other ordering, and applying a similar reasoning, we get an overall factor of 4 . Thus, combining with results from section 2.1, we get, for holomorphic primary fields

$$
-\frac{\mu_{3}}{4}\left\langle\left\{W_{3}, \phi_{k}(w)\right\}\right\rangle_{\mathrm{str}}^{\mathrm{conn}}=-\mu_{3}\left\langle W_{3} \phi_{k}(w)\right\rangle_{\mathrm{cyl}}^{\mathrm{conn}}
$$

A similar statement is true for an antiholomorphic primary field.

Let us turn now to primary fields $\phi_{k}(w, \bar{w})$ with $h_{k}, \bar{h}_{k} \neq 0$ (of the form $\phi_{k}(w, \bar{w})=$ $\varphi_{k}(w) \varphi_{k}(\bar{w})$, as discussed before in the context of (2.4)). In the cylinder calculation in section 2.1 the $\mu$-corrections for these vanished. In the present case, they are non-zero for operators of the form $\phi_{k}(w, \bar{w})=\varphi_{k}(w) \bar{\varphi}_{k}(\bar{w})$, with $h_{k}=\bar{h}_{k}$ (as in (2.3)). After conformally transforming to the UHP, we regard $\bar{\varphi}_{k}$ on the UHP as $\varphi_{k}^{*}$ at the image point on the LHP (up to a constant). Combining with the arguments used for the holomorphic operators, we eventually get

$$
\begin{aligned}
\frac{\left\langle\left\{W_{3}, \phi_{k}(w, \bar{w})\right\}\right\rangle_{\mathrm{str}}^{\mathrm{conn}}}{\left\langle\phi_{k}(w, \bar{w})\right\rangle_{\mathrm{str}}} & =i^{3} \frac{2 \pi}{\beta^{2}}(z \bar{z})^{h} I_{3}\left(z, z^{\prime}\right), \\
I_{3}\left(z, z^{\prime}\right) & \equiv \int_{\Gamma_{1}+\Gamma_{1}^{\prime}+\tilde{\Gamma}_{1}+\tilde{\Gamma}_{1}^{\prime}} \underset{z_{1}^{2}\left\langle\mathcal{W}_{3}\left(z_{1}\right) \varphi_{k}(z) \varphi_{k}^{*}\left(z^{\prime}\right)\right\rangle_{\mathbb{C}}^{\text {conn }} /\left\langle\varphi_{k}(z) \varphi_{k}^{*}\left(z^{\prime}\right)\right\rangle_{\mathbb{C}}^{\text {conn }}}{ }
\end{aligned}
$$

The ratio of correlators inside the integral is given by

$$
\left\langle\mathcal{W}_{3}\left(z_{1}\right) \varphi_{k}(z) \varphi_{k}^{*}\left(z^{\prime}\right)\right\rangle_{\mathbb{C}}^{\text {conn }} /\left\langle\varphi_{k}(z) \varphi_{k}^{*}\left(z^{\prime}\right)\right\rangle_{\mathbb{C}}^{\text {conn }}=q_{3} \frac{\left(z-z^{\prime}\right)^{3}}{\left(z_{1}-z\right)^{3}\left(z_{1}-z^{\prime}\right)^{3}}
$$

where $q_{3}$ is the $\mathcal{W}_{3}$-charge of the field $\phi_{k}$. Integrals of the kind (2.20) are discussed in detail in appendix A.2. The final result (see (A.6)) is that the $O\left(\mu_{3}\right)$ correction, in the long time limit (2.6), is given by (using that all four contours $\Gamma_{1}, \tilde{\Gamma}_{1}, \Gamma_{1}^{\prime}, \tilde{\Gamma}_{1}^{\prime}$ contribute equally, 
cancelling the $1 / 4$ in $-\mu_{3} / 4$ )

$$
\begin{aligned}
\left\langle\phi_{k}(\sigma, t)\right\rangle_{\mathrm{dyn}} & =a_{k} e^{-2 \pi \Delta_{k} t / \beta}\left(1-Q_{3, k} \tilde{\mu}_{3}\left(\frac{2 \pi t}{\beta}+\mathrm{constant}\right)+O\left(\mu_{3}^{2}\right)\right)+\ldots, \\
Q_{3, k} & =i^{3} 2 q_{3, k}(2 \pi), \quad \tilde{\mu}_{3}=\frac{\mu_{3}}{\beta^{2}}, \quad \Delta_{k}=2 h_{k}
\end{aligned}
$$

Up to $O\left(\mu_{3}\right)$, it agrees with (1.7).

In case of a quasiprimary field $\phi_{k}(w, \bar{w})$, it mixes, under conformal transformation to the plane, with lower dimension operators. The most relevant operator among these, which is of the form $\varphi_{k}(z) \varphi_{k}(\bar{z})$, is then to be used in (2.20) for obtaining the dominant timedependence; in that case $\Delta_{k}, Q_{3, k}$ refer to this operator (rather than to the original $\phi_{k}$ ).

For higher $W_{n}$ charges, the currents $\mathcal{W}_{n}(w)$ are typically quasiprimary, and hence they mix with lower order $\mathcal{W}_{m}(z)$ under conformal transformation to the UHP. Thus the $O\left(\mu_{n}\right)$ correction to the dynamical one-point function $\left\langle\phi_{k}\right\rangle_{\text {dyn }}$ is a linear combination of terms of the form (A.3) (weighted by a set of coefficients $a_{n, m}$, as in (2.23) below). Collecting all this, the $O(\mu)$ correction with all chemical potentials is given by

$$
\begin{aligned}
\left\langle\phi_{k}(\sigma, t)\right\rangle_{\mathrm{dyn}} & =a_{k} e^{-2 \pi \Delta_{k} t / \beta}\left(1-\sum_{n=3} Q_{n, k} \tilde{\mu}_{n}\left(\frac{2 \pi t}{\beta}+\text { constant }\right)+O\left(\mu^{2}\right)\right)+\ldots, \\
\tilde{\mu}_{n} & =\frac{\mu_{n}}{\beta^{n-1}}, \quad \Delta_{k}=h_{k}+\bar{h}_{k}=2 h_{k} \\
Q_{n, k} & =2 \sum_{m=0}^{\lfloor n / 2-1\rfloor} a_{n, m} i^{n-2 m}(2 \pi)^{n-2 m-2} q_{n-2 m, k} \\
& =i^{n}(2 \pi)^{n-2} 2 q_{n, k}+i^{n-2}(2 \pi)^{n-4} a_{n, 2} 2 q_{n-2, k}+\ldots,
\end{aligned}
$$

Note that for $W_{3}$ deformations, the expression for $Q_{3}$ as in (2.22) corresponds only to the first term in the above series expression for $Q_{n}$. This is because the $\mathcal{W}_{3}$ current is a primary field and does not mix with any lower $\mathcal{W}$ current under a conformal transformation. From $n=4$ onwards, the additional terms in $Q_{n, k}$ 's represent the mixing of $\mathcal{W}_{n}$ currents with $\mathcal{W}_{n-2 m}$ under conformal transformations.

\subsubsection{Higher order $\mu$-corrections}

Let us first consider that $O\left(\mu_{n}^{2}\right)$ correction:

$$
\left.\left\langle\phi_{k}(w, \bar{w})\right\rangle_{\mathrm{str}}^{\mathrm{conn}}\right|_{2} ^{\mu_{n}} \equiv \frac{\left(\mu_{n} / 4\right)^{2}}{2 !}\left(\left\langle\left\{W_{n} W_{n}, \phi_{k}(w, \bar{w})\right\}\right\rangle_{\mathrm{str}}^{\mathrm{conn}}+2\left\langle W_{n} \phi_{k}(w, \bar{w}) W_{n}\right\rangle_{\mathrm{str}}^{\mathrm{conn}}\right)
$$

Again, for holomorphic (or antiholomorphic) primary fields $\phi_{k}(w)$, it is straightforward to generalize (2.19) to this order.

$$
\left.\left\langle\phi_{k}(w)\right\rangle_{\mathrm{str}}^{\mathrm{conn}}\right|_{2} ^{\mu_{n}}=\frac{\mu_{n}^{2}}{2 !}\left\langle W_{n} W_{n} \phi_{k}(w)\right\rangle_{\mathrm{cyl}}^{\mathrm{conn}}
$$

For a primary field of the form $\phi_{k}(w, \bar{w})=\varphi_{k}(w) \varphi_{k}(\bar{w})$, proceeding as in the previous subsection, we get

$$
\left.\left\langle\phi_{k}(w)\right\rangle_{\mathrm{str}}^{\mathrm{conn}}\right|_{2} ^{\mu_{n}}=\frac{1}{2 !}\left(Q_{n, k} \tilde{\mu}_{n} t \frac{2 \pi}{\beta}\right)^{2}+\mu_{n}^{2}(\text { constant } \times t+\text { constant })+\ldots
$$


The essential ingredient in this calculation is

$$
\begin{aligned}
I_{n m}\left(z, z^{\prime} \mid \Gamma_{1}, \Gamma_{2}\right) & \equiv \int_{\Gamma_{1}} d z_{1} z_{1}^{n-1} \int_{\Gamma_{2}} d z_{2} z_{2}^{m-1} f_{n m}\left(z_{1}, z_{2}, z, z^{\prime}\right) \\
f_{n m}\left(z_{1}, z_{2}, z, z^{\prime}\right) & =\frac{\left\langle\mathcal{W}_{n}\left(z_{1}\right) \mathcal{W}_{m}\left(z_{2}\right) \varphi_{k}(z) \varphi_{k}^{*}\left(z^{\prime}\right)\right\rangle_{\mathbb{C}}^{\text {conn }}}{\left\langle\varphi_{k}(z) \varphi_{k}^{*}\left(z^{\prime}\right)\right\rangle_{\mathbb{C}}^{\text {conn }}}
\end{aligned}
$$

By repeating the strategy of (A.10), we get

$$
\begin{aligned}
& \text { Coefficient of }\left[\log \left(-z^{\prime}\right)-\log (-z)\right]^{2} \text { in } I_{n m}\left(z, z^{\prime} \mid \Gamma_{1}, \Gamma_{2}\right) \\
& \quad=\text { Residue }_{z_{1}=z}\left[\operatorname{Residue~}_{z_{2}=z}\left(\frac{\left\langle\mathcal{W}_{n}\left(z_{1}\right) \mathcal{W}_{m}\left(z_{2}\right) \varphi_{k}(z) \varphi_{k}^{*}\left(z^{\prime}\right)\right\rangle_{\mathbb{C}}^{\text {conn }}}{\left\langle\varphi_{k}(z) \varphi_{k}^{*}\left(z^{\prime}\right)\right\rangle_{\mathbb{C}}^{\text {conn }}}\right)\right]=q_{n, k} q_{m, k}
\end{aligned}
$$

where we have first used the $\mathcal{W}_{m}\left(z_{2}\right) \varphi_{k}(z)$ OPE, and then the $\mathcal{W}_{n}\left(z_{1}\right) \varphi_{k}(z)$ OPE. In a manner similar to that in appendix A.2, we conclude the following structure of $I_{n m}\left(z, z^{\prime}\right)$ :

$$
\begin{aligned}
I_{n m}\left(z, z^{\prime} \mid \Gamma_{1}, \Gamma_{2}\right)=q_{n, k} q_{m, k}( & {\left.\left[\log \left(-z^{\prime}\right)-\log (-z)\right]+\text { constant }\right) } \\
\times & \left(\left[\log \left(-z^{\prime}\right)-\log (-z)\right]+\text { constant }\right)
\end{aligned}
$$

Note that at late times $t \gg \beta,\left(\left[\log \left(-z^{\prime}\right)-\log (-z)\right] \rightarrow 2(2 \pi t) / \beta\right.$ and dominates over the constant term (the precise sense is that of (2.34)). Similar to appendix A.2, the $4 \times 4=16$ locations of the contour-pairs $\left(\Gamma_{1}, \Gamma_{2}\right),\left(\Gamma_{1}, \Gamma_{2}^{\prime}\right),\left(\Gamma_{1}, \tilde{\Gamma}_{2}\right),\left(\Gamma_{1}, \tilde{\Gamma}_{2}^{\prime}\right), \ldots$, all contribute equally, therefore converting $\left(\mu_{n} / 4\right)\left(\mu_{m} / 4\right) \rightarrow \mu_{n} \mu_{m}$. Combining all these, we get (2.26). The charges $q_{n}$ that are defined by the $\mathcal{W}_{n} \varphi$ OPE and appear in (2.28), get multiplied by some constants $^{12}$ and shifted by lower $\mathcal{W}_{n-2 k}$ charges to give the $Q_{n}$ in (2.28), as in (2.23).

Arbitrary orders and exponentiation: it is straightforward to generalize the above $O\left(\tilde{\mu}^{2}\right)$ calculation to higher orders in the perturbation in chemical potentials. Thus, at the order $\prod_{i=1}^{r} \mu_{n_{i}}$, there are $r$ insertions of $\mathcal{W}$-currents, leading to integrals of the form

$$
\begin{aligned}
& I_{n_{1} n_{2} \ldots n_{r}}\left(z, z^{\prime} \mid \Gamma_{1}, \Gamma_{2}, \ldots, \Gamma_{r}\right) \\
& \quad \equiv \int_{\Gamma_{1}} d z_{1} z_{1}^{n_{1}-1} \int_{\Gamma_{2}} d z_{2} z_{2}^{n_{2}-1} \ldots \int_{\Gamma_{r}} d z_{2} z_{r}^{n_{r}-1} f_{n_{1} n_{2} \ldots n_{r}}\left(z_{1}, z_{2}, \ldots, z_{r} ; z, z^{\prime}\right), \\
& f_{n_{1} n_{2} \ldots n_{r}}\left(z_{1}, z_{2}, \ldots, z_{r} ; z, z^{\prime}\right) \\
& \quad=\frac{\left\langle\mathcal{W}_{n_{1}}\left(z_{1}\right) \mathcal{W}_{n_{2}}\left(z_{2}\right) \ldots \mathcal{W}_{n_{r}}\left(z_{r}\right) \varphi_{k}(z) \varphi_{k}^{*}\left(z^{\prime}\right)\right\rangle_{\mathbb{C}}^{\operatorname{conn}}}{\left\langle\varphi_{k}(z) \varphi_{k}^{*}\left(z^{\prime}\right)\right\rangle_{\mathbb{C}}^{\operatorname{conn}}}
\end{aligned}
$$

\footnotetext{
${ }^{12} \operatorname{Each} \mathcal{W}_{n}$ current comes with a factor of $\frac{i^{n}}{2 \pi}\left(\frac{2 \pi}{\beta}\right)^{n-1}$, as in (2.9).
} 
Again, repeating the strategy of (A.10), we get the following leading $\left(\right.$ viz. $\left.(\log )^{r}\right)$ contribution (see (2.34) for the definition of the leading-log contribution)

$$
\begin{aligned}
\text { Coefficient of }\left[\log \left(-z^{\prime}\right)-\log (-z)\right]^{r} \text { in } I_{n_{1} n_{2} \ldots n_{r}}\left(z, z^{\prime} \mid \Gamma_{1}, \Gamma_{2}, \ldots, \Gamma_{r}\right) \\
=\text { Residue }_{z_{1}=z}\left[\ldots \text { Residue }_{z_{r-1}=z}\right. \\
\left.\quad\left\{\operatorname{Residue}_{z_{r}=z}\left(\frac{\left\langle\mathcal{W}_{n_{1}}\left(z_{1}\right) \ldots \mathcal{W}_{n_{r-1}}\left(z_{r-1}\right) \mathcal{W}_{n_{r}}\left(z_{r}\right) \varphi_{k}(z) \varphi_{k}^{*}\left(z^{\prime}\right)\right\rangle_{\mathbb{C}}^{\text {conn }}}{\left\langle\varphi_{k}(z) \varphi_{k}^{*}\left(z^{\prime}\right)\right\rangle_{\mathbb{C}}^{\text {conn }}}\right)\right\}\right] \\
=q_{n_{1}, k} \ldots q_{n_{r-1}, k} q_{n_{r}, k}
\end{aligned}
$$

where we have first used the $\mathcal{W}_{n_{r}}\left(z_{r}\right) \varphi_{k}(z)$ OPE, then $\mathcal{W}_{n_{r-1}}\left(z_{r-1}\right) \varphi_{k}(z)$ OPE, etc. As in the $O\left(\mu^{2}\right)$ calculation above, we obtain the following behaviour at late times

$$
\begin{aligned}
I_{n_{1} n_{2} \ldots n_{r}}\left(z, z^{\prime} \mid \Gamma_{1}, \Gamma_{2}, \ldots, \Gamma_{r}\right) & \\
=q_{n_{1}, k} \ldots q_{n_{r-1}, k} q_{n_{r}, k} & \underbrace{\begin{array}{r}
\left.\left[\log \left(-z^{\prime}\right)-\log (-z)\right]+\text { constant }\right) \\
\times \ldots \times\left(\left[\log \left(-z^{\prime}\right)-\log (-z)\right]+\text { constant }\right)
\end{array}}_{r \text { terms }}
\end{aligned}
$$

The two equations above show that the leading log contribution to (2.30) from every contour integral of the $\mathcal{W}_{n_{i}}$ current contributes the factor $q_{n_{i}}\left[\log \left(-z^{\prime}\right)-\log (-z)\right]$. This is the first basic ingredient for the exponentiation we are going to find. Furthermore, it is easy to see that the leading $\log$ contribution is the same irrespective of where each contour $\Gamma_{i}$ is placed (out of 4 possible choices, e.g. $\Gamma_{1}, \Gamma_{1}^{\prime}, \tilde{\Gamma}_{1}, \tilde{\Gamma}_{1}^{\prime}$ in figure 1 ). As before we must combine the contribution of all positions of the contours, which, therefore, amounts to multiplying the result for (2.30) by $4^{r}$ which converts the original coefficients coming from $\exp \left[-\sum_{n} \mu_{n} W_{n} / 4\right]$ as follows

$$
\frac{\prod_{i=1}^{r} \mu_{i} / 4}{r !} \rightarrow \frac{\prod_{i=1}^{r} \mu_{i}}{r !}
$$

This is the second basic factor leading to the exponentiation. Combining all these, and incorporating some additional constants (see footnote 12) we get the following, leading, order $\left(\mu_{n_{1}} \ldots \mu_{n_{r}}\right)$ contribution

$$
\left.\left\langle\phi_{k}(w)\right\rangle_{\mathrm{str}}^{\mathrm{conn}}\right|_{r} ^{\mu_{n_{1}} \ldots \mu_{n_{r}}}=\frac{1}{r !} \prod_{i=1}^{r}\left(Q_{n_{i}, k} \tilde{\mu}_{n_{i}} \frac{2 \pi}{\beta}\right)+O\left(\mu^{r} t^{r-l}\right)
$$

Once again, the constants $Q_{n}$ are related to the $q_{n}$ as in (2.23)) in a manner similar to the $O(\tilde{\mu})$ and the $O\left(\tilde{\mu}^{2}\right)$ calculation above. We note that the leading log contribution used in this paper can be isolated by considering a scaling

$$
\tilde{\mu}_{n} \rightarrow 0, \tilde{t} \equiv \frac{t}{\beta} \rightarrow \infty, \text { such that } \tilde{\mu}_{n} \tilde{t}=\text { constant. }
$$

The second term in (2.33), or for that matter, in (2.26), is subleading at large times in the sense of this scaling. 


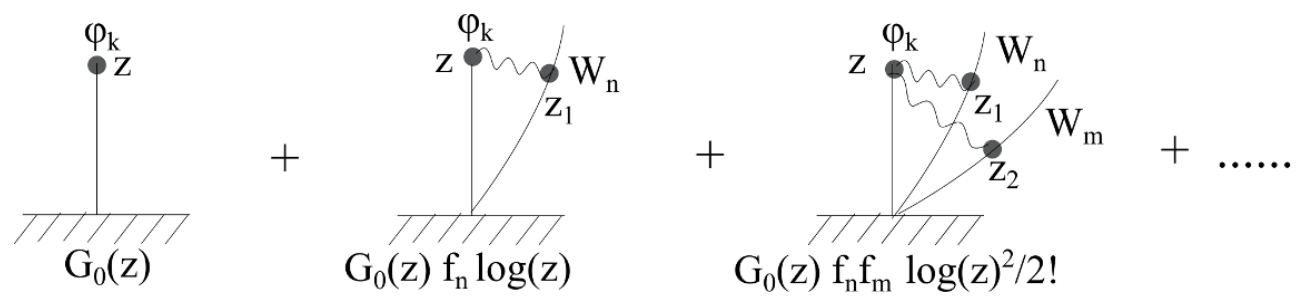

Figure 2. The schematics of the calculation of the one-point function. The first term represents the zero-order boundary Green's function (2.3) without chemical potentials (the shading indicates the boundary of the upper half plane). The second term represents the $O\left(\mu_{n}\right)$ correction, which involves one insertion of a $W_{n}$-charge (which is an integral over the $z_{1}$-contour. As explained in the text, at long times, this insertion amounts to multiplying the zero order term by a term of the form $f_{n} \log (z)$, where $f_{n}$ is described in (2.23). The third term represents insertion of two such $W$-charges; as we explained in the text (see (2.26) and below), each insertion again amounts to multiplying by the factor mentioned above, along with a factor of $\frac{1}{2 !}$. The pattern continues, to ensure an exponentiation to $G_{0}(z) z^{\sum_{n} f_{n}}$, as in (2.35). Since at long times $G_{0}(z) \sim e^{-\gamma_{k}^{(0)} t}$ (see $(2.3)$ ), and $z \sim e^{-2 \pi t / \beta}$, adding the chemical potentials amount to a shift of the exponent $\gamma_{k}^{(0)} \rightarrow \gamma_{k}$ as in (1.6).

Using the above results, we now have, for primary fields of the form $\phi_{k}(w, \bar{w})=$ $\varphi_{k}(w) \varphi_{k}(\bar{w})$

$$
\begin{aligned}
\left\langle\phi_{k}(w, \bar{w})\right\rangle_{\text {str }}= & a_{k} e^{-\frac{2 \pi \Delta_{k} t}{\beta}}\left[1-\sum_{n} \tilde{\mu}_{n} Q_{n, k}\left(\frac{2 \pi t}{\beta}+\text { const }\right)\right. \\
& +\frac{1}{2 !} \sum_{n, m} \tilde{\mu}_{n} \tilde{\mu}_{m} Q_{n, k}\left(\frac{2 \pi t}{\beta}+\text { const }\right) Q_{m, k}\left(\frac{2 \pi t}{\beta}+\text { const }\right)+\ldots \\
& \left.+\frac{1}{r !} \sum_{\left\{n_{i}\right\}} \prod_{i=1}^{r} \tilde{\mu}_{n_{i}} Q_{n_{i}, k}(\underbrace{\left(\frac{2 \pi t}{\beta}+\text { const }\right) \ldots\left(\frac{2 \pi t}{\beta}+\text { const }\right)}_{r \text { terms }})+\ldots\right] \\
= & a_{k} e^{-2 \pi t / \beta\left(\Delta_{k}+\sum_{n} \tilde{\mu}_{n} Q_{n, k}+O\left(\tilde{\mu}^{2}\right)\right)}=a_{k} e^{-\gamma_{k} t}
\end{aligned}
$$

where we have absorbed some constant factors in $a_{k} . \gamma_{k}$ is given by (1.7); $Q_{n, k}$ are the shifted $W_{n}$ charges of $\phi_{k}$ as defined in (2.23). The proof of the above equation for general quasiprimary operators $\phi_{k}(w, \bar{w})$ works out much the same way as in case of the $O(\mu)$ terms, as discussed in section 2.2.1. We emphasize that it is only the leading contributions at large times which we have proved here to exponentiate. Thus, we do not claim that the constant terms marked "const" in the above equation are all the same. As we have remarked before, the leading contributions can be isolated using the scaling mentioned in (2.34).

The schematics of the above calculation is explained in the figure 2.

\section{Calculation of $I(t)$}

Let us rewrite the expression for the thermalization function $I(t)(1.8)$ in the form

$$
I(t)=Z_{s c} / \sqrt{Z_{s s} Z_{c c}}=\hat{Z}_{s c} / \sqrt{\hat{Z}_{s s} \hat{Z}_{c c}},
$$




$$
\begin{aligned}
Z_{s c} & \equiv \operatorname{Tr}\left(\rho_{\mathrm{dyn}, A}(t) \rho_{\mathrm{eqm}, A}(\beta, \mu)\right), & & \hat{Z}_{s c}=Z_{s c} /\left(Z_{s} Z_{c}\right) \\
Z_{s s} & \equiv \operatorname{Tr}\left(\rho_{\mathrm{dyn}, A}(t) \rho_{\mathrm{dyn}, A}(t)\right), & & \hat{Z}_{s s}=Z_{s s} / Z_{s}^{2}, \\
Z_{c c} & \equiv \operatorname{Tr}\left(\rho_{\mathrm{eqm}, A}(\beta, \mu) \rho_{\mathrm{eqm}, A}(\beta, \mu)\right), & & \hat{Z}_{c c}=Z_{c c} / Z_{c}^{2}, \\
Z_{s} & =\operatorname{Tr}\left(\rho_{\mathrm{dyn}}(t)\right)=\left\langle\psi_{0} \mid \psi_{0}\right\rangle, & & Z_{c}=\operatorname{Tr}\left(\rho_{\beta, \mu}\right)
\end{aligned}
$$

In appendix B we explain how to compute $I(t)$ using the short interval expansion, valid when the length of the interval $l$ is small compared with the other time scales $\beta$ and $t$ in the problem. We reproduce the main formula (B.4) for our purpose, where we explicitly denote the dependencies on the length $l$ of the interval, the inverse temperature $\beta$ and the chemical potentials $\mu$ (the dependence on $\beta$ on the r.h.s. is implicit; the one-point functions depend on both $\beta$ and $\mu$ - see section 2).

$$
\begin{aligned}
& \hat{Z}_{s c}(l, \beta, \mu)=\sum_{k_{1}, k_{2}} C_{k_{1}, k_{2}}(l)\left\langle\phi_{k_{1}}\left(w_{1}, \bar{w}_{1}\right)\right\rangle_{\mathrm{str}}^{\mu}\left\langle\phi_{k_{2}}\left(w_{2}, \bar{w}_{2}\right)\right\rangle_{\mathrm{cyl}}^{\mu}, \\
& \hat{Z}_{s s}(l, \beta, \mu)=\sum_{k_{1}, k_{2}} C_{k_{1}, k_{2}}(l)\left\langle\phi_{k_{1}}\left(w_{1}, \bar{w}_{1}\right)\right\rangle_{\mathrm{str}}^{\mu}\left\langle\phi_{k_{2}}\left(w_{2}, \bar{w}_{2}\right)\right\rangle_{\mathrm{str}}^{\mu}, \\
& \hat{Z}_{c c}(l, \beta, \mu)=\sum_{k_{1}, k_{2}} C_{k_{1}, k_{2}}(l)\left\langle\phi_{k_{1}}\left(w_{1}, \bar{w}_{1}\right)\right\rangle_{\mathrm{cyl}}^{\mu}\left\langle\phi_{k_{2}}\left(w_{2}, \bar{w}_{2}\right)\right\rangle_{\mathrm{cyl}}^{\mu}
\end{aligned}
$$

It is understood, for the logic of the short interval expansion to go through, that all contours which represent insertion of the $W$-charges (see figure 1) are drawn outside of the small disc-like region of both sheets of figure 4 .

\subsection{Proof of thermalization}

Using the short-interval expansion above, and the long time behaviour of one-point functions from section 2), it is easy to prove that the system thermalizes in the sense of (1.10) or $(1.11)$.

To prove this, note that it is only the holomorphic (or antiholomorphic) fields $\phi_{k}$ which possibly have non-zero expectation values in the long time limit (2.6). For these fields, the one-point functions on the cylinder and on the strip agree (see (2.7), (2.19), (2.25) ). By virtue of (3.2), we therefore have in the long time limit $Z_{s c}=Z_{s s}=Z_{c c}$. Hence using the expression (3.1) for the thermalization function we get $I(t \rightarrow \infty)=1$ which proves (1.10) and consequently (1.11).

The above-mentioned equality of one-point functions between the strip and cylinder geometries for holomorphic (or antiholomorphic) fields imply the same for the conserved $\mathcal{W}_{n^{-}}$(or $\overline{\mathcal{W}}_{n}$ )- currents. This, therefore, proves that

$$
\left\langle\psi(t)\left|W_{n}\right| \psi(t)\right\rangle=\operatorname{Tr}\left(W_{n} \rho_{\text {eqm }}\right)
$$

Note that in proving this, we have used the correspondence (1.4) between the parameters of the initial state and the putative equilibrium state. The above equation, therefore, proves the correspondence (1.4). 


\subsection{Thermalization rate}

To evaluate the rate of approach of $I(t)$ to its asymptotic value 1, we organize the terms in $\hat{Z}_{s c}, \hat{Z}_{s s}, \hat{Z}_{c c}$ as follows

$$
\begin{array}{rlrl}
\hat{Z}_{s c} & =C_{0,0}\left(1+S_{1}^{s c}\right), & S_{1}^{s c} & =\sum_{a} \hat{C}_{a, 0}\left(\left\langle\phi_{a}\right\rangle_{\mathrm{str}}^{\mu}+\left\langle\phi_{a}\right\rangle_{\mathrm{cyl}}^{\mu}\right)+\sum_{a b} \hat{C}_{a, b}\left\langle\phi_{a}\right\rangle_{\mathrm{str}}^{\mu}\left\langle\phi_{b}\right\rangle_{\mathrm{cyl}}^{\mu} \\
\hat{Z}_{s s}=C_{0,0}\left(1+S_{1}^{s s}+S_{2}^{s s}\right), & S_{1}^{s s} & =2 \sum_{a} \hat{C}_{a, 0}\left\langle\phi_{a}\right\rangle_{\mathrm{str}}^{\mu}+\sum_{a b} \hat{C}_{a, b}\left\langle\phi_{a}\right\rangle_{\mathrm{str}}^{\mu}\left\langle\phi_{b}\right\rangle_{\mathrm{str}}^{\mu} \\
S_{2}^{s s} & =\sum_{k} \hat{C}_{k, k}\left(\left\langle\phi_{k}\right\rangle_{\mathrm{str}}^{\mu}\right)^{2} \\
\hat{Z}_{c c} & =C_{0,0}\left(1+S_{1}^{c c}\right), & S_{1}^{c c} & =2 \sum_{a} \hat{C}_{a, 0}\left\langle\phi_{a}\right\rangle_{\mathrm{cyl}}^{\mu}+\sum_{a b} \hat{C}_{a, b}\left\langle\phi_{a}\right\rangle_{\mathrm{cyl}}^{\mu}\left\langle\phi_{b}\right\rangle_{\mathrm{cyl}}^{\mu}
\end{array}
$$

where $a, b, \ldots$ denote descendents of the identity operator, $k$ labels other primaries (than the identity) and their descendents. $\hat{C} \equiv C / C_{0,0}$.

\subsection{1 $\mu=0$}

Let us first consider the case of zero chemical potentials. Using the results in sections 2, and appendices A and B.1, we get

$$
\begin{aligned}
S_{1}^{s c} & =-a_{T} \tilde{l}^{2}\left(1+O(\tilde{l})^{2}\right)+a_{T \bar{T}^{4}} \tilde{l}^{4} e^{-8 \pi t / \beta}\left(1+O(\tilde{l})^{2}\right)+O\left(e^{-8 \pi \tilde{t}}\right) \\
S_{1}^{s s} & =-a_{T} \tilde{l}^{2}\left(1+O(\tilde{l})^{2}\right)+2 a_{T \bar{T}} \tilde{l}^{4} e^{-8 \pi \tilde{t}}\left(1+O(\tilde{l})^{2}\right)+O\left(e^{-8 \pi \tilde{t}}\right) \\
S_{2}^{s s} & =\sum_{k}\left[a_{k} \tilde{l}^{4 h_{k}} e^{-8 \pi h_{k} t / \beta}\left(1+O(\tilde{l})^{2}\right)+O\left(e^{-12 \pi h_{k} \tilde{t}}\right)\right] \\
S_{1}^{c c} & =-a_{T} \tilde{l}^{2}\left(1+O(\tilde{l})^{2}\right) \\
a_{T} & =\frac{c \pi^{2}}{24}, \quad a_{T \bar{T}}=\frac{A_{T \bar{T}} \pi^{4}}{8 c} \quad a_{k}=\frac{A_{k}^{2}}{n_{k}}\left(\frac{\pi}{2}\right)^{4 h_{k}}
\end{aligned}
$$

To this order, it is easy to see that the contribution to $I(t)$ from descendents of identity, demarcated by $a_{T}, a_{T \bar{T}}$, vanishes. The leading contribution to $I(t)$, demarcated by $a_{k}$, occurs only in $\hat{Z}_{s s}$ and comes from $\left(\left\langle\phi_{m}(z, \bar{z})\right\rangle_{\text {str }}\right)^{2}$ for which $h_{k}$ is the minimum $\left(=h_{m}\right)$ (this could be a field which appears after a conformal transformation of the original quasiprimary field). The time-dependence shown of $S_{2}^{s s}$ comes from (2.3). Using this, we get

$$
I(t)=1-\alpha \exp \left[-2 \gamma_{m}^{(0)} t\right]+\ldots, \gamma_{m}^{(0)}=2 \pi \Delta_{m} / \beta
$$

This is of the form (1.9) for $\mu=0$, with

$$
\alpha \equiv \frac{A_{m}^{2}}{n_{m}}\left(\frac{\pi}{2}\right)^{4 h_{m}}(\tilde{l})^{4 h_{m}}\left(1+O(\tilde{l})^{2}\right)
$$

The discarded terms in (3.6) are faster transients. This proves (1.9) for zero chemical potential. This result has already appeared in [32]. ${ }^{13}$

\footnotetext{
${ }^{13}$ Our exponent differs from Cardy's value by a factor of 2 .
} 


\subsection{2 $\mu \neq 0$}

The generalization of the above result to the case of non-zero chemical potentials is straightforward. Once again, the dominant time-dependence arises from $\left(\left\langle\phi_{m}(z, \bar{z})\right\rangle_{\mathrm{str}}^{\mu}\right)^{2}$ in the $S_{2}^{s s}$ or $\hat{Z}_{s s}$. The time-dependence (1.9) follows by using (2.35) in $S_{2}^{s s}$.

\subsection{Properties of $\hat{Q}$}

From the asymptotic behaviour (1.9) of the thermalization function we indicated the asymptotic behaviour (1.13) of the dynamical reduced density matrix $\hat{\rho}_{\mathrm{dyn}}(t)$. By using the long time behaviour of the one-point functions (1.6), we can easily deduce the following dominant behaviour of overlaps of $\hat{Q}$ with various quasiprimary fields at late times

$$
\operatorname{Tr}\left(\hat{Q} \phi_{k}(t)\right) \propto e^{-\left(\gamma_{k}-\gamma_{m}\right) t}, \operatorname{Tr}\left(\hat{Q} \phi_{m}(t)\right) \rightarrow \text { constant. }
$$

\section{Decay of perturbations of a thermal state}

We found in the previous sections that the long time behaviour of the reduced density matrix $\rho_{\mathrm{dyn}, A}(t)$ resembles that of a thermal ensemble plus a small deformation which decays exponentially. We will find in the next section that the thermal ensemble (or more accurately the generalized Gibbs ensemble) corresponds to a (higher spin) black hole geometry in the bulk. The small perturbation of the equilibrium ensemble is thus expected to correspond to a small deformation of the black hole geometry. Consequently, the exponential decay of the deformation in the CFT should correspond to a 'ringing-down' or a quasinormal mode in the bulk.

We will address the above issue in the next section which deals with bulk geometry. However, in order to make the correspondence of the above paragraph more precise, in this section we will directly present a CFT computation of the decay of a perturbation to a thermal state. Note that this computation is, in principle, different from the exponential decay of the one-point function in the quenched state, (1.6). However, what we will find is that the long time behaviour (1.6) of an operator $\phi_{k}(0, t)$ in the quenched state is the same as that of its two-point function (4.1) in the thermal state (1.3) (with chemical potentials). The latter measures the thermal decay of a perturbation and is more directly related to a black hole quasinormal mode. Throughout this section, we will assume that the conformal dimensions of $\phi_{k}$ satisfy $h_{k}=\bar{h}_{k}$.

We define the thermal two-point function as ${ }^{14}$

$$
G_{+}(t, 0 ; \beta, \mu) \equiv \frac{1}{Z} \operatorname{Tr}\left(\phi_{k}(0, t) \phi_{k}(0,0) e^{-\beta H-\sum_{n} \mu_{n} W_{n}}\right)
$$

By the techniques developed in the earlier sections, a computation of this quantity amounts to calculating the following correlator on the plane

$$
\left\langle\phi_{k}(z, \bar{z}) \phi_{k}(y, \bar{y}) e^{-\sum_{n} \mu_{n} W_{n}}\right\rangle, \quad z=i e^{-2 \pi t / \beta}, \bar{z}=-i e^{2 \pi t / \beta}, y=i, \bar{y}=-i
$$

\footnotetext{
${ }^{14}$ We use the same notations as in [45].
} 


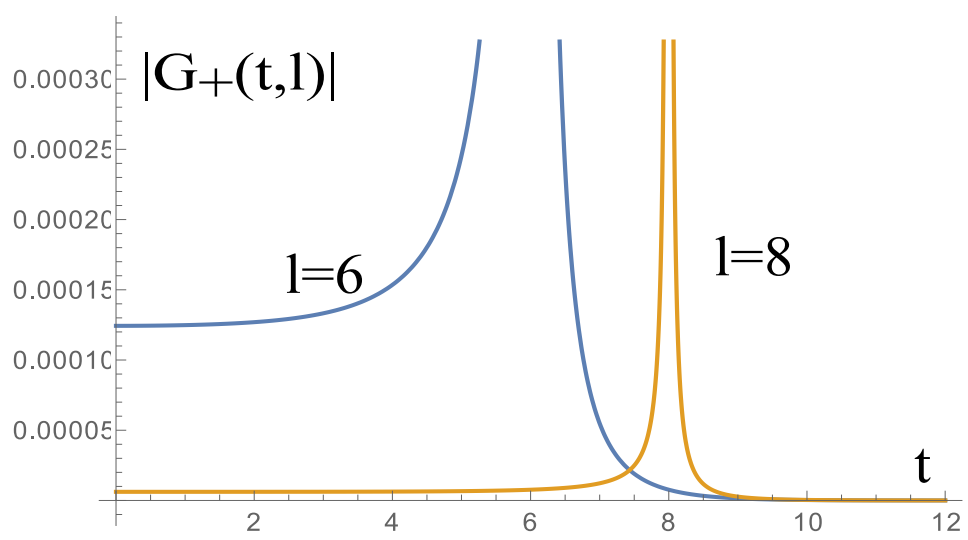

Figure 3. Plots of the thermal Green's function $G_{+}(t, l ; \beta, 0)$ for $\beta=2 \pi, \Delta_{k}=1.5$. The curve on the left (blue) is for $l=6$, and the curve on the right (orange) is for $l=8$. Note that the exponential decay in time occurs for times larger than $l$.

where the $\mu_{n}$-deformations are understood as an infinite series of contours as in the previous section.

For $\mu=0$, the above two-point function is standard. Including the Jacobian of transformation, we get

$$
G_{+}(t, 0 ; \beta, 0)=\left(\frac{2 \pi}{\beta}\right)^{4 h_{k}}\left[\left(i e^{-2 \pi t / \beta}-i\right)\left(-i e^{2 \pi t / \beta}+i\right)\right]^{-2 h_{k}} \stackrel{t \rightarrow \infty}{\longrightarrow} \text { const } e^{-2 \pi t \Delta_{k} / \beta},
$$

which clearly matches the long time behaviour of the one-point function (1.6) in the quenched state for $\mu=0$. Here $\Delta_{k}=2 h_{k}$.

In the above, we considered the thermal Green's function for two points which are both at the same spatial point $\sigma=0$. It is easy to compute the Green's function when the two points are spatially separated by a distance $l$, say with $\sigma_{1}=l$ and $\sigma_{2}=0$. We get

$$
\begin{aligned}
& G_{+}(t, l ; \beta, 0) \equiv \frac{1}{Z} \operatorname{Tr}\left(\phi_{k}(l, t) \phi_{k}(0,0) e^{-\beta H}\right) \\
&= {\left[\frac{2 \pi}{\beta} e^{\pi l / \beta}\right]^{4 h_{k}}\left(\left(i e^{2 \pi(l-t) / \beta}-i\right)\left(-i e^{2 \pi(l+t) / \beta}+i\right)\right)^{-2 h_{k}} } \\
& \stackrel{t, l \gg \beta}{\longrightarrow}\left\{\begin{array}{l}
\text { const } e^{-2 \pi t \Delta_{k} / \beta},(t-l) \gg \beta \\
\text { const } e^{-2 \pi l \Delta_{k} / \beta},(l-t) \gg \beta
\end{array}\right.
\end{aligned}
$$

The coordinates of the two points, in the notation of (4.2) are modified here to $z=$ $i e^{2 \pi(l-t) / \beta}, \bar{z}=-i e^{2 \pi(l+t) / \beta}, y=i, \bar{y}=-i$. The prefactor with the square bracket comes from the Jacobian of the transformation from the cylinder to the plane. The behaviour of the Green's function is shown in figure 3. It is important to note that the exponential decay, found in (1.6) shows up only for time scales $t \gg l$.

The effect of turning on the chemical potentials can be dealt with as in the previous sections. At $O\left(\mu_{n}\right)$, we will have, as before, a holomorphic contribution and an antiholomorphic contribution. The former is proportional to

$$
\left\langle\phi_{k}(\bar{z}) \phi_{k}(\bar{y})\right\rangle \times \int_{\Gamma} d z_{1} z_{1}^{n-1}\left\langle\mathcal{W}_{n}\left(z_{1}\right) \phi_{k}(z) \phi_{k}(y)\right\rangle
$$


As we see, the structure of the integral is the same as in the previous section. As before, logarithmic terms appear in the above integrals which give the leading, linear, $t$-dependence. Similar remarks also apply to the antiholomorphic contour. Since the calculations are very similar to those in the previous two sections, we do not provide all details. By resumming the series over the infinite number of contours, we find in a straightforward fashion that

$$
G_{+}(t, 0 ; \beta, \mu) \stackrel{t \rightarrow \infty}{\longrightarrow} G_{+}(0,0 ; \beta, 0) b(\mu) e^{-\gamma_{k} t}
$$

where $b(\mu)$ is time-independent, and is of the form $b(\mu)=1+O(\mu)$. This long time decay is the same as that of the one-point function (1.6) in the quenched state, as claimed above. For points separated by a distance $l$, the above exponential decay shows up for $t \gg l$, as in (4.4).

In the above, we have discussed the two-point function in real space. It is straightforward to convert the result (4.4) without chemical potentials to Fourier space, which develops poles at

$$
\left.\omega_{k, m}\right|_{\mu=0}=-i \frac{2 \pi}{\beta}\left(\Delta_{k}+2 m\right), m=0,1,2, \ldots
$$

Our results in (1.6) can be interpreted as a shift, caused by the presence of the chemical potentials $\mu_{n}$, of the dominant pole $\left.\omega_{k, 0}\right|_{\mu=0}$ to

$$
\omega_{k, 0}=-i \frac{2 \pi}{\beta}\left(\Delta_{k}+\sum_{n} \tilde{\mu}_{n} Q_{n, k}\right)=-i \gamma_{k}
$$

where the notation is the same as that of (1.6). In this paper we will not address the question of the shift of the subdominant poles $\omega_{k, m}$ (for $m=1,2, \ldots$ ) due to chemical potentials (the current status of these can be found in $[26,27,46]$ ).

Two-point functions of the kind (4.1), for a single chemical potential $\mu_{3}$, and up to order $\mu_{3}^{2}$, have appeared earlier in [26] (calculations up to $O\left(\mu_{3}^{5}\right)$ have appeared in [27]). What we find in our paper is that at large times, the perturbation series in $\mu_{n}$, up to all orders in all chemical potentials, can be resummed, to yield the leading correction to the thermalization rate in the presence of chemical potentials.

At a technical level, the one-point function in the quenched state corresponds to a onepoint function in a geometry with a boundary, and for operators considered here, these turn into a two-point function on the plane, by virtue of the method of images. The thermal decay naturally involves a two-point function on the plane ${ }^{15}$ and agrees with the above two-point function at late times.

\section{$5 \quad$ Holography and higher spin black holes}

Zero chemical potential: as remarked in the Introduction, a global quantum quench described by an initial state of the form (1.5), for large central charges and zero chemical

\footnotetext{
${ }^{15}$ Actually the thermal calculation involves a product of two such factors, one holomorphic and the other antiholomorphic, but one of the factors just gives an overall constant and only one factor leads to the important time-dependence.
} 
potentials, has been shown in [34-36] to be dual to one half of the eternal BTZ (black string) geometry, whose boundary represents an end-of-the-world brane.

In an independent development, it was found in [47] that the quasinormal mode of a scalar field $\Phi_{k}(\sigma, t, z)$ of mass $m$ in a BTZ background (dual to a CFT operator $\phi_{k}$ of dimension $\left.\Delta_{k} \equiv 1+\sqrt{1+m^{2}}\right)$ is of the form $\exp [-2 \pi \Delta t / \beta]$ at large times. This time-dependence agrees with the CFT exponent in (4.4) exactly. This shows that the exponential decay of a CFT perturbation to a thermal state corresponds to the decay of the corresponding scalar field in the bulk geometry. This result has been extended to higher spin fields in the BTZ background in [48].

Non-zero chemical potentials: in case the CFT has additional conserved charges, in particular if it has a representation of a $W_{\infty}$ algebra (and consequently the hs $(\lambda)$ algebra [37]), then the bulk dual corresponding to those conserved charges have been conjectured to be the conserved higher spin charges of higher spin gravity. In particular, [38, 39] have shown that if one interprets the grand canonical ensemble (1.4) (more generally, the GGE) in the framework of an $h s(\lambda)$ representation, then the bulk dual corresponds to a higher spin black hole.

Thus, we would like to conjecture that the bulk dual of the quantum quench with chemical potentials, would correspond to a gravitational collapse to a higher spin black hole.

As an important consistency check, by analogy with the case with zero potential, in the present case too, the leading quasinormal mode (QNM) of a scalar field $\Phi_{k}(\sigma, t, z)$ should have a time-dependence given by (4.6). Following the results in [46] (see also $[26,27,40])^{16}$ we find that at late times $t \gg \beta$ the QNM for the hs $(\lambda)$ scalar field $\Phi_{+}$behaves, up to $O\left(\mu_{3}\right)$, as $e^{-i \omega_{k, 0} t}$, where

$$
\omega_{k, 0}=-i \frac{2 \pi}{\beta}\left(1+\lambda+\tilde{\mu}_{3} \frac{1}{3}(1+\lambda)(2+\lambda)\right)
$$

where the index $k$ here refers to the operator $\phi_{k}$ dual to the scalar field $\Phi_{+}$. Noting that for this operator we have $\Delta_{k}=1+\lambda$, and $Q_{3, k}=\frac{1}{3}(1+\lambda)(2+\lambda)[26,27]$, we see that the QNM frequency $\omega_{k, 0}$ agrees, to the relevant order, with the pole (4.8) of the thermal 2-point function which, in turn, is related to the thermalization exponent by the relation $\omega_{k, 0}=-i \gamma_{k}$, with $\gamma_{k}$ given in (1.6).

\section{Discussion}

In this paper, we considered quantum quench to a critical point which is described by a 2D CFT with additional conserved charges besides the energy. We used a generalized ansatz (1.1) for the quenched state which is obtained by regulating a conformal boundary state with multiple cut-off parameters. ${ }^{17}$ It was found that local observables in such a

\footnotetext{
${ }^{16}$ We wish to thank Alejandro Cabo-Bizet and Viktor Giraldo-Rivera for informing us that the difference between equation (5.1) above and the corresponding equation (4.2) in a previous version of their paper [46] was due to a typo, which has now been corrected in the new version of their paper.

${ }^{17}$ As explained in footnote 1, the ansatz (1.1) generalizes the Calabrese-Cardy (CC) state. It is possible to verify, for specific quench protocols in simple integrable theories [31], that the quenched state actually
} 
state thermalize to an equilibrium described by a grand canonical ensemble (1.4) with temperature and chemical potentials related to the cut-off parameters. We computed the thermalization rate for various observables, including the reduced density matrix for an interval. It was found that the same rate appears also in the long time decay of two-point functions in equilibrium (see (1.6) and (1.14)).

In the context where the number of conserved charges is infinite, so that the CFT is integrable, our results prove that thermalization is generic to all integrable CFTs, provided that the charges are derived from local conserved currents and that the spectrum of conformal dimensions has a gap. By contrast, to our knowledge, there is no such general proof of thermalization for integrable models with mass gaps (although a large number of examples are known, see page 3). It is interesting to mention that these models typically have a power-law relaxation to the GGE (see, e.g. $[21-23]),{ }^{18}$ in contrast with the exponentially fast relaxation found in this paper for the conformal case.

We found that for integrable models, the reduced density matrix reduces to that in an equilibrium ensemble (a generalized Gibbs ensemble, GGE) which holographically corresponds to a higher spin black hole $[38,39]$. We found that the thermalization rate found above agrees with the leading quasinormal frequency of the higher spin black hole; this constitutes an additional, dynamical, evidence for the holographic correspondence between the global quenches in this paper and the evolution into the higher spin black hole.

One of the main technical advances made in this paper is the resummation of leading$\log$ terms at large times, presented in section 2.2.2, which leads to exponentiation of the perturbation series, leading to the thermalization rate, presented in (1.6), (2.35), as a function of chemical potentials. This allows us to also compute the effect of chemical potentials on the relaxation times of thermal Green's functions. Another technical advance consists of the computation of the long-time reduced density matrix (1.9), using a short-interval expansion, which allows us to prove thermalization of an arbitrary string of local observables.

As we mentioned above, our ansatz of the initial state (1.1) for the post-quench evolution accommodates additional details of the quench procedure in terms of the additional conserved charges (see footnotes 1 and 17). We expect that the results we obtain regarding equilibration and relaxation rates, when expressed in terms of the conserved charges, will be general, irrespective of the specific choice of the initial state. We should also mention that the effect of the higher $W_{n}$ charges, which are higher dimension operators, is subdominant at low energies (as is clear from the expression of the thermalization exponents). In general, we would expect that a generic quenched state would correspond to deforming the boundary state by a set of irrelevant operators; however, a generic irrelevant operator which is not associated with additional conserved charges is expected not to modify the final equilibrium ensemble. We hope to return to this important question in the near future.

takes the form (1.1). In case of a sudden quench which is characterized by a single scale, e.g. the initial mass gap, the parameters $\epsilon_{n}$ are all related; whereas for a more general quench protocol with multiple scales, our generalization of the $\mathrm{CC}$ state accommodates the additional scales.

${ }^{18}$ The power-law relaxation in these works can be understood in terms of a non-relativistic dispersion relation of quasiparticles. In case of a critical quench, the quasiparticles have a relativistic dispersion relation, leading to an exponential decay. 
One might wonder whether the results presented in this paper are tied to the use of translationally invariant quenched states such as (1.1), whose energy density and various charge densities are uniform. We will address the question of inhomogeneous quench in a forthcoming paper [31], both in the CFT and in the holographic dual, using the methods of [49] where we create an inhomogeneous energy density by applying conformal transformations. It turns out [31] that if the initial state has inhomogeneities in a compact domain and has uniform energy densities outside, local observables again thermalize asymptotically with exponents governed by the uniform densities. Other important issues involve local quenches (see, e.g. $[50,51]$ ), and compact spatial dimensions. The issue of thermalization when space is compact is quite subtle. It has been shown in [32] that at large times one can have the phenomenon of revival (observables effectively returning to their initial values). The dynamical entanglement entropy for a quantum quench in a space with boundaries is an interesting, related, issue; we hope to come back to this in a forthcoming publication [52].

\section{Acknowledgement}

We would like to thank Pallab Basu, Justin David, Deepak Dhar, Oleg Evnin, Rajesh Gopakumar, Shiraz Minwalla, Pranjal Nayak, Arunabha Saha, David Simmons-Duffin and Tomonori Ugajin for discussions and Somyadip Thakur for discussions and collaboration in the forthcoming paper [40], partial results from which are presented here. We would also like to thank Juan Pedraza for drawing our attention to ref. [5] and Alejandro Cabo-Bizet and Viktor Giraldo-Rivera for a useful correspondence regarding ref. [46]. We would also like to thank the referee for drawing our attention to important references and discussions in the literature.

\section{A Some details on one-point functions}

Here we collect some additional helpful material on the one-point functions discussed in this paper.

\section{A.1 A few explicit one-point functions with zero chemical potentials}

Case $\boldsymbol{k}=$ descendent of identity: in this case, $\phi_{k}(w, \bar{w})$ is of the form $T, \bar{T}$, or $: T \bar{T}$ : or some descendents thereof. Under a conformal transformation (2.2), these operators pick up a c-number term in addition to a term proportional to the corresponding operator on the plane/UHP. We will give some examples to illustrate the calculation

1. Cylinder: in this case

$$
\begin{aligned}
\langle T(w)\rangle_{\mathrm{cyl}} & =\left\langle\left(-\frac{c \pi^{2}}{6 \beta^{2}}-\frac{4 \pi^{2}}{\beta^{2}} z^{2} T(z)\right)\right\rangle_{U H P}=-\frac{c \pi^{2}}{6 \beta^{2}} \\
\langle: T \bar{T}:(w, \bar{w})\rangle_{\mathrm{cyl}} & =\left\langle\left(\left[-\frac{c \pi^{2}}{6 \beta^{2}}-\frac{4 \pi^{2}}{\beta^{2}} z^{2} T(z)\right]\left[-\frac{c \pi^{2}}{6 \beta^{2}}-\frac{4 \pi^{2}}{\beta^{2}} \bar{z}^{2} \bar{T}(\bar{z})\right]\right)\right\rangle_{U H P} \\
& =\left(\frac{c \pi^{2}}{6 \beta^{2}}\right)^{2}
\end{aligned}
$$


2. Strip: in this case

$$
\begin{aligned}
\langle T(w)\rangle_{\mathrm{str}} & =\left\langle\left(-\frac{c \pi^{2}}{6 \beta^{2}}-\frac{4 \pi^{2}}{\beta^{2}} z^{2} T(z)\right)\right\rangle_{U H P}=-\frac{c \pi^{2}}{6 \beta^{2}}=\langle T(w)\rangle_{\mathrm{cyl}} \\
\langle: T \bar{T}:(w, \bar{w})\rangle_{\mathrm{str}} & =\left\langle\left(\left[-\frac{c \pi^{2}}{6 \beta^{2}}-\frac{4 \pi^{2}}{\beta^{2}} z^{2} T(z)\right]\left[-\frac{c \pi^{2}}{6 \beta^{2}}-\frac{4 \pi^{2}}{\beta^{2}} \bar{z}^{2} \bar{T}(\bar{z})\right]\right)\right\rangle_{U H P} \\
& =\left(\frac{c \pi^{2}}{6 \beta^{2}}\right)^{2}+A_{T \bar{T}}(z-\bar{z})^{-4}=\left(\frac{c \pi^{2}}{6 \beta^{2}}\right)^{2}+a_{T \bar{T}} e^{-8 \pi t / \beta}+\ldots
\end{aligned}
$$

where $A_{T \bar{T}}, a_{T \bar{T}}$ are constants as in (2.3) and (2.4).

Case $k=$ descendent of other primaries: in this case,

1. cylinder: the one-point function vanishes as in the case of primaries.

2. strip: the one-point function can be related to one-point function of primaries which is dealt with above.

\section{A.2 Some details on $O\left(\mu_{n}\right)$ correction to the one-point function}

In this section we will consider the following integrals which arise in connection with $O\left(\mu_{n}\right)$ correction to the one-point function $\langle\phi(\sigma, t)\rangle_{\mathrm{dyn}}$ :

$$
\begin{aligned}
I_{n}\left(z, z^{\prime} \mid \Gamma_{1}\right) & \equiv \int_{\Gamma_{1}} d z_{1} z_{1}^{n-1} f_{n}\left(z_{1}, z, z^{\prime}\right), \quad g_{n}\left(z_{1}, z, z^{\prime}\right) \equiv \int d z_{1} z_{1}^{n-1} f_{n}\left(z_{1}, z, z^{\prime}\right) \\
f_{n}\left(z_{1}, z, z^{\prime}\right) & =\frac{\left\langle\mathcal{W}_{n}\left(z_{1}\right) \varphi_{k}(z) \varphi_{k}^{*}\left(z^{\prime}\right)\right\rangle_{\mathbb{C}}^{\operatorname{conn}}}{\left\langle\varphi_{k}(z) \varphi_{k}^{*}\left(z^{\prime}\right)\right\rangle_{\mathbb{C}}^{\operatorname{conn}}}=q_{n, k} \frac{\left(z-z^{\prime}\right)^{n}}{\left(z_{1}-z\right)^{n}\left(z_{1}-z^{\prime}\right)^{n}}
\end{aligned}
$$

The second integral on the first line is an indefinite integral. The integrals above can be explicitly computed. E.g.

$$
\begin{aligned}
g_{3}\left(z_{1}, z, z^{\prime}\right) & =q_{3, k}\left[R_{3}\left(z, z^{\prime}\right)\left(\log \left(z_{1}-z\right)-\log \left(z_{1}-z^{\prime}\right)\right)-\frac{z^{2}}{2\left(z_{1}-z\right)^{2}}+\frac{z^{\prime 2}}{2\left(z_{1}-z^{\prime}\right)^{2}}\right. \\
\left.+\frac{z^{\prime}\left(2 z+z^{\prime}\right)}{\left(z-z^{\prime}\right)\left(z_{1}-z^{\prime}\right)}+\frac{z\left(2 z^{\prime}+z\right)}{\left(z-z^{\prime}\right)\left(z_{1}-z\right)}\right] & \\
I_{3}\left(z, z^{\prime} \mid \Gamma_{1}\right) & =q_{3, k}\left[R_{3}\left(z, z^{\prime}\right)\left(-\log (-z)+\log \left(-z^{\prime}\right)\right)+3 \frac{\left(z+z^{\prime}\right)}{\left(z-z^{\prime}\right)}\right] \\
R_{3}\left(z, z^{\prime}\right) & \equiv \frac{\left(z^{2}+4 z z^{\prime}+z^{\prime 2}\right)}{\left(z-z^{\prime}\right)^{2}}
\end{aligned}
$$

Note that $I_{3}$ is essentially obtained from the lower limit of the integral, i.e. from $-g\left(0, z, z^{\prime}\right)$. The contour $\Gamma_{1}$ in $I_{3}$ specifies which branch of the log is to be taken. In particular

$$
I_{3}\left(z, z^{\prime} \mid \Gamma_{1}\right)-I_{3}\left(z, z^{\prime} \mid \tilde{\Gamma}_{1}\right)=-2 \pi i q_{3, k} R_{3}\left(z, z^{\prime}\right)
$$

In the long time limit (2.6), we get

$$
I_{3}\left(z, z^{\prime} \mid \Gamma_{1}\right)=I_{3}\left(z, z^{\prime} \mid \tilde{\Gamma}_{1}\right)=2 q_{3, k} t(2 \pi / \beta)+q_{3, k} \times \mathrm{const}+O\left(e^{-2 \pi t / \beta}\right)
$$


In this equation we have displayed the principal value of the relevant integrals (the discontinuity (A.5) tells us the coefficient of the log term or the linear $t$ term).

However, we would like to understand the above results more simply, by using the $\mathcal{W}_{n}\left(z_{1}\right) \varphi_{k}(z)$ OPE which is of the form:

$$
\mathcal{W}_{n}\left(z_{1}\right) \varphi_{k}(z)=q_{n, k} \frac{\varphi_{k}(z)}{\left(z_{1}-z\right)^{n}}+\sum_{i=1}^{n-1} \alpha_{n, i} \frac{\varphi_{k, i}(z)}{\left(z_{1}-z\right)^{n-i}}+\text { regular terms }
$$

where $\varphi_{k, i}(z)$ is of dimension $h_{k}+i{ }^{19}$ Using this, we get an expansion for the connected 3 -point function of the form:

$$
\frac{\left\langle\mathcal{W}_{n}\left(z_{1}\right) \varphi_{k}(z) \varphi_{k}^{*}\left(z^{\prime}\right)\right\rangle_{\mathbb{C}}^{\text {conn }}}{\left\langle\varphi_{k}(z) \varphi_{k}^{*}\left(z^{\prime}\right)\right\rangle_{\mathbb{C}}^{\text {conn }}}=\frac{q_{n, k}}{\left(z_{1}-z\right)^{n}}+\frac{C_{n, 1}}{\left(z_{1}-z\right)^{n-1}\left(z-z^{\prime}\right)}+O\left(z-z^{\prime}\right)^{-2}
$$

Performing the integral in (A.3),

$$
\begin{aligned}
g_{n}\left(z_{1}, z, z^{\prime}\right)=q_{n, k}( & \left.\log \left[z_{1}-z\right]-(n-1) \frac{z}{z_{1}-z}+\ldots\right) \\
& +\frac{C_{n, 1}}{z-z^{\prime}}\left(z_{1}-z+(n-1) z \log \left[z_{1}-z\right]+\ldots\right)+\ldots
\end{aligned}
$$

The ellipsis in each round bracket represents terms with higher powers of $1 /\left(z_{1}-z\right.$ ) (up to a maximum of $\left.\left(z_{1}-z\right)^{-n}\right)$; successive round brackets themselves are arranged in higher inverse powers of $z-z^{\prime}$. Using the $\mathcal{W}_{n}\left(z_{1}\right) \varphi_{k}^{*}\left(z^{\prime}\right)$ OPE in a similar fashion and using the symmetry property $g_{n}\left(z_{1}, z, z^{\prime}\right)=(-1)^{n} g_{n}\left(z_{1}, z^{\prime}, z\right)$ we can arrive at a general structure

$$
g_{n}\left(0, z, z^{\prime}\right)=q_{n, k}\left(\log [-z]-\log \left[-z^{\prime}\right]\right) R_{n}\left(z, z^{\prime}\right)+\ldots
$$

where $R_{n}\left(z, z^{\prime}\right)=(-1)^{n-1} R_{n}\left(z^{\prime}, z\right)$ is of the form $P_{n-1}\left(z, z^{\prime}\right) /\left(z-z^{\prime}\right)^{n-1}\left(P_{n-1}\left(z, z^{\prime}\right)\right.$ is a homogeneous symmetric polynomial of degree zero). See the explicit form of $R_{n}$ for $n=3$ in (A.4). The omitted terms are all ratios of homogeneous polynomials in $\left(z, z^{\prime}\right)$ of the same degree in the numerator and in the denominator. This implies that we have, in the long time limit (2.6)

$$
I_{n}\left(z, z^{\prime} \mid \Gamma_{1}\right)=I_{3}\left(z, z^{\prime} \mid \tilde{\Gamma}_{1}\right)=2 q_{n, k}(2 \pi / \beta) t+q_{n, k} \times \text { const }+O\left(e^{-2 \pi t / \beta}\right)
$$

which, of course, agrees with (A.6).

Note that the dominant time-dependence $2 q_{n, k} t(2 \pi / \beta)$ comes from the long-time limit of the coefficient $R_{n}\left(z, z^{\prime}\right)$ of the log terms, which can be read off from the discontinuity $I_{n}\left(z, z^{\prime} \mid \Gamma_{1}\right)-I_{n}\left(z, z^{\prime} \mid \tilde{\Gamma}_{1}\right)$ (see (A.5)). Now, the contour $\int_{\Gamma_{1}-\tilde{\Gamma}_{1}} d z_{1}$ can be deformed to a very small circle $\oint \Gamma_{z} d z_{1}$ around the point $z$; therefore the leading long-time behaviour $R_{n}^{(0)}\left(z, z^{\prime}\right)$ can be derived by using the leading OPE singularity in (A.7) and computing the residue at $z_{1}=z$ :

Coefficient of $\left[\log \left(-z^{\prime}\right)-\log (-z)\right]$ in $I_{n}\left(z, z^{\prime}\right)$

$$
=\text { Residue }_{z_{1}=z}\left(\frac{\left\langle\mathcal{W}_{n}\left(z_{1}\right) \varphi_{k}(z) \varphi_{k}^{*}\left(z^{\prime}\right)\right\rangle_{\mathbb{C}}^{\text {conn }}}{\left\langle\varphi_{k}(z) \varphi_{k}^{*}\left(z^{\prime}\right)\right\rangle_{\mathbb{C}}^{\text {conn }}}\right) \equiv q_{n, k} R_{n}^{(0)}\left(z, z^{\prime}\right)=q_{n, k}
$$

\footnotetext{
${ }^{19}$ This is the general form; some of the $\alpha_{n, i}$ coefficients may, of course, vanish.
} 


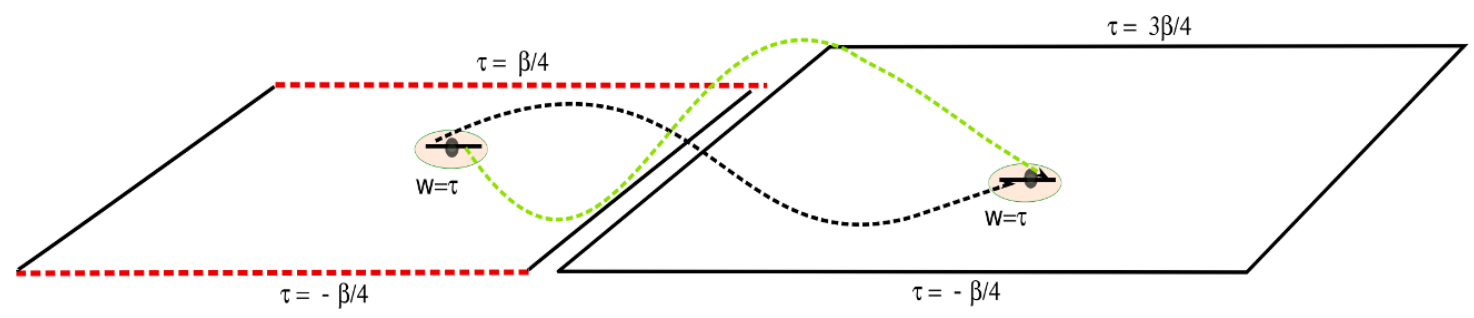

Figure 4. Two different geometries, the strip and the cylinder, glued along the cut as described in the text. The method of the short interval expansion allows us to compute the functional integral over this geometry by replacing a small tube enclosing the two glued cuts by a complete basis of operators $\phi_{k_{1}} \otimes \phi_{k_{2}}$ where the operators live in the two Hilbert spaces.

\section{B Short interval expansion}

In this section we will explain a formalism suitable for computing partition functions of the kind that appear in (3.1). For convenience we will first compute these quantities in Euclidean time $\tau=i t$ and later analytically continue back to Lorentzian time. With this, each of the expressions $Z_{s c}, Z_{s s}, Z_{c c}$ is of the form

$$
\operatorname{Tr}\left(\rho_{A, 1} \rho_{A, 2}\right)=\int_{\text {geometry } 1} \mathbf{D} \varphi_{1} \underset{\text { geometry } 2}{\mathbf{D} \varphi_{2}} \delta\left(F\left[\varphi_{1}, \varphi_{2}\right]\right) \exp \left(-S\left[\varphi_{1}\right]-S\left[\varphi_{2}\right]\right)
$$

where $S[\varphi]$ represents the action for the CFT (with fields $\varphi$ ) and the delta-functional in the measure represents a gluing condition between a geometry ' 1 ' and a geometry ' 2 ' along a 'cut' which is the location, at a particular time $\tau$, of the spatial interval $A: \sigma \in$ $(-l / 2, l / 2) .{ }^{20}$ For $Z_{s s}$, both geometries are that of a strip of the Euclidean plane described by complex coordinates $(w, \bar{w})=\sigma \pm i \tau$ defined by boundaries at $\tau= \pm \beta / 4$ with boundary conditions determined by the boundary state $|B d\rangle$ introduced in (1.5). For $Z_{c c}$, both geometries are that of a cylinder cut of the Euclidean plane with identified boundaries at $\tau=-\beta / 4,3 \beta / 4$. The geometries for both $Z_{s s}$ and $Z_{c c}$ are familiar from calculations of Entanglement Renyi entropy (of order 2) and can be calculated from appropriate correlation functions of twist fields [53] which exchange two identical geometries. For $Z_{s c}$, the two glued geometries are different (that of a strip and a cylinder), hence the method of twist operators do not apply in a straightforward fashion. (See figure 4). In this paper, we will therefore, employ the method of the short interval expansion.

The idea of the short interval expansion [54] is as follows. To begin, we express the functional integral (B.1) as an overlap of two wavefunctions in $\mathrm{H}_{1} \otimes \mathrm{H}_{2}$, as follows

$$
\begin{aligned}
& Z_{12}=\operatorname{Tr}\left(\rho_{A, 1} \rho_{A, 2}\right)=\left\langle\psi_{\text {out }} \mid \psi_{\text {in }}\right\rangle=\int_{w_{1} \in \mathcal{D}_{1}} \mathbf{D} \bar{\varphi}_{1}\left(w_{1}\right) \int_{w_{2} \in \mathcal{D}_{2}} \mathbf{D} \bar{\varphi}_{2}\left(w_{2}\right) \psi_{\text {in }}\left[\overline{\varphi_{1}}, \overline{\varphi_{2}}\right] \psi_{\text {out }}^{*}\left[\overline{\varphi_{1}}, \overline{\varphi_{2}}\right] \\
& \psi_{\text {in }}\left[\overline{\varphi_{1}}, \overline{\varphi_{2}}\right] \equiv \int_{w_{1} \in \mathcal{D}_{1}}^{\mathbf{D} \varphi_{1}\left(w_{1}\right)} \int_{w_{2} \in \mathcal{D}_{2}}^{\mathbf{D} \varphi_{2}\left(w_{2}\right)} \delta\left(\left.\varphi_{1}\right|_{\partial \mathcal{D}_{1}}-\overline{\varphi_{1}}\right) \\
& \times \delta\left(\left.\varphi_{2}\right|_{\partial \mathcal{D}_{2}}-\overline{\varphi_{2}}\right) \delta\left(F\left[\varphi_{1}, \varphi_{2}\right]\right) \exp \left(-S\left[\varphi_{1}\right]-S\left[\varphi_{2}\right]\right)
\end{aligned}
$$

\footnotetext{
${ }^{20}$ To be precise, $\delta[F]=\delta\left(\varphi_{1}\left(A_{<}\right)-\varphi_{2}\left(A_{>}\right)\right) \delta\left(\varphi_{1}\left(A_{>}\right)-\varphi_{2}\left(A_{<}\right)\right)$, where $A_{<}\left(A_{>}\right)$represents the limiting value from below (above) the cut.
} 


$$
\psi_{\text {out }}\left[\overline{\varphi_{1}}, \overline{\varphi_{2}}\right] \equiv \int_{w_{1} \notin \mathcal{D}_{1}} \mathbf{D} \varphi_{1}\left(w_{1}\right) \int_{w_{2} \notin \mathcal{D}_{2}}^{\mathbf{D} \varphi_{2}}\left(w_{2}\right) \delta\left(\left.\varphi_{1}\right|_{\partial \mathcal{D}_{1}}-\overline{\varphi_{1}}\right) \delta\left(\left.\varphi_{2}\right|_{\partial \mathcal{D}_{2}}-\overline{\varphi_{2}}\right) \exp \left(-S\left[\varphi_{1}\right]-S\left[\varphi_{2}\right]\right)
$$

Here $\mathcal{D}_{1}$ (respectively, $\mathcal{D}_{2}$ ) is a small disc drawn around the cut in geometry 1 (respectively, geometry 2).

Note that only $\left|\psi_{\text {in }}\right\rangle$ depends on the gluing condition since the delta functional in the measure does not affect $\left|\psi_{\text {out }}\right\rangle$. The basic point of the short interval is that in the limit when the length $l$ of the cut is small compared with the characterizing length scale of the geometries (in our case, when $l \ll \beta$ ), the wavefunction $\psi_{\text {in }}\left[\varphi_{1}, \varphi_{2}\right]$ becomes jointly localized at the centre $\left(w_{1}, \bar{w}_{1}\right)$ of the disc $\mathcal{D}_{1}$ and at the centre $\left(w_{2}, \bar{w}_{2}\right)$ of the disc $\mathcal{D}_{2},{ }^{21}$ and hence can be expanded in terms of local operators, as follows

$$
\left|\psi_{\text {in }}\right\rangle=\sum_{k_{1}, k_{2}} C_{k_{1}, k_{2}} \phi_{k_{1}}\left(w_{1}, \bar{w}_{1}\right) \phi_{k_{2}}\left(w_{2}, \bar{w}_{2}\right)|0\rangle_{1} \otimes|0\rangle_{2}
$$

Here $k_{1}, k_{2}$ label a complete basis of quasiprimary operators of the CFT Hilbert space. Each term in the sum represents a factorized wavefunction (between geometries 1 and 2), which, therefore, gives ${ }^{22}$

$$
\begin{aligned}
& \hat{Z}_{s c}=\sum_{k_{1}, k_{2}} C_{k_{1}, k_{2}}\left\langle\phi_{k_{1}}\left(w_{1}, \bar{w}_{1}\right)\right\rangle_{\mathrm{str}}\left\langle\phi_{k_{2}}\left(w_{2}, \bar{w}_{2}\right)\right\rangle_{\mathrm{cyl}}, \\
& \hat{Z}_{s s}=\sum_{k_{1}, k_{2}} C_{k_{1}, k_{2}}\left\langle\phi_{k_{1}}\left(w_{1}, \bar{w}_{1}\right)\right\rangle_{\mathrm{str}}\left\langle\phi_{k_{2}}\left(w_{2}, \bar{w}_{2}\right)\right\rangle_{\mathrm{str}}, \\
& \hat{Z}_{c c}=\sum_{k_{1}, k_{2}} C_{k_{1}, k_{2}}\left\langle\phi_{k_{1}}\left(w_{1}, \bar{w}_{1}\right)\right\rangle_{\mathrm{cyl}}\left\langle\phi_{k_{2}}\left(w_{2}, \bar{w}_{2}\right)\right\rangle_{\mathrm{cyl}}
\end{aligned}
$$

Here the subscripts str and cyl refer to "strip", and "cylinder" respectively. The one-point functions are evaluated on the respective geometries without any cut (see section 2 for more details). The glued functional integral (B.1), (B.2) is recovered by summing over $k_{1}, k_{2}$ with the coefficients $C_{k_{1}, k_{2}}$; , as clear from (B.4) these are determined by the gluing condition and depend on the size of the cut [54] (see section B.1 for more details).

\section{B.1 The coefficients $C_{k_{1}, k_{2}}$}

As explained in [54] (see also section B), the coefficients $C_{k_{1}, k_{2}}$ are determined by the equation

$$
C_{k_{1}, k_{2}}=\frac{Z_{2}}{Z_{1}^{2}}\left(n_{k_{1}} n_{k_{2}}\right)^{-\frac{1}{2}} \lim _{z_{1} \rightarrow \infty_{1}, z_{2} \rightarrow \infty_{2}}\left(z_{1} z_{2}\right)^{2\left(h_{k_{1}}+h_{k_{2}}\right)}\left(\bar{z}_{1} \bar{z}_{2}\right)^{2\left(\bar{h}_{k_{1}}+\bar{h}_{k_{2}}\right)}\left\langle\phi_{k_{1}}\left(z_{1}, \bar{z}_{1}\right) \phi_{k_{2}}\left(z_{2}, \bar{z}_{2}\right)\right\rangle_{\mathbb{C}_{2}}
$$

where $\mathbb{C}_{2}$ represents two infinite planes glued along a cut $A, Z_{2}$ is the functional integral such a glued geometry and $Z_{1}$ is the functional integral over a single plane. This equation

\footnotetext{
${ }^{21}$ We will take the centre of the disc in each geometry to coincide with the centre of the cut, which has coordinates $w=i \tau, \bar{w}=-i \tau$.

${ }^{22}$ In case geometries 1 and 2 are identical, the superscripts in $w_{i}, \bar{w}_{i}, i=1,2$ indicate which sheet we are considering.
} 
can be easily proved by inserting quasiprimary a operator at infinity in each plane in an equation like (B.1) or (B.2). The two point function in the glued geometry is to be determined by using the uniformizing map:

$$
y=\sqrt{(z+l / 2) /(z-l / 2)}
$$

The normalization constants $n_{k}$ are determined by the following orthogonality condition of the quasiprimary operators

$$
\left\langle\phi_{k_{1}}\left(z_{1}, \bar{z}_{1}\right) \phi_{k_{2}}\left(z_{2}, \bar{z}_{2}\right)\right\rangle_{\mathbb{C}}=\frac{n_{k_{1}} \delta_{k_{1}, k_{2}}}{z_{12}^{h_{k_{1}}+h_{k_{2}}} \bar{z}_{k_{1}+\bar{h}_{k_{2}}}}
$$

where $n_{k_{1}}$ is a normalization constant. Note that $C_{k_{1}, k_{2}}=C_{k_{2}, k_{1}}$. Below we will use the notation

$$
\hat{C}_{k_{1}, k_{2}}=C_{k_{1}, k_{2}} / C_{0,0}
$$

Case $\left(\boldsymbol{k}_{1}, \boldsymbol{k}_{\mathbf{2}}\right)=(\mathbf{0}, \mathbf{0})$ : we will denote the identity operator as $\phi_{0}=1$. It is obvious that

$$
C_{0,0}=Z_{2} / Z_{1}^{2}
$$

Case $\left(\boldsymbol{k}_{1}, \boldsymbol{k}_{2}\right)=(\boldsymbol{k}, \mathbf{0})$ : the only case where $C_{k, 0} \neq 0$ is when $\phi_{k}(z, \bar{z})$ is a descendent of the identity operator, e.g. $T(z), \bar{T}(\bar{z}),: T(z) \bar{T}(\bar{z}):, \Lambda(z), \Lambda(\bar{z})$ etc. $^{23}$ E.g.

$$
\hat{C}_{T, 0}=C_{T, 0} / C_{0,0}=\hat{C}_{\bar{T}, 0}=\frac{l^{2}}{16} ; \hat{C}_{T \bar{T}, 0}=\frac{l^{4}}{256} ; \ldots
$$

All other $C_{k, 0}$ vanish as they are proportional to a one-point function of a primary operator on the Riemann surface (and hence to that on the complex plane).

Case $\left(k_{1}, k_{2}\right)=$ (primary, primary): in case $\phi_{k_{1}}, \phi_{k_{2}}$ are primary operators, (B.5) gives

$$
\hat{C}_{k_{1}, k_{2}}=\frac{1}{n_{k_{1}}} \delta_{k_{1}, k_{2}}\left(\frac{l e^{i \pi / 2}}{4}\right)^{2\left(h_{k_{1}}+\bar{h}_{k_{1}}\right)}
$$

Case $\left(k_{1}, k_{2}\right)=$ (descendent, descendent): in case $\phi_{k_{1}}$ is of the form $L_{-n_{1}} L_{-n_{2}} \ldots \bar{L}_{-m_{1}} \bar{L}_{-m_{2}} \ldots \phi_{l_{1}}$ and $\phi_{k_{2}}$ is of the form $L_{-r_{1}} L_{-r_{2}} \ldots \bar{L}_{-s_{1}} \bar{L}_{-s_{2}} \ldots \phi_{l_{2}}$, we can show that

$$
\begin{aligned}
\hat{C}_{k_{1}, k_{2}} & =\delta_{l_{1}, l_{2}} \delta_{\sum n, \sum r} \delta_{\sum m, \sum s} A\left(n_{1}, n_{2}, \ldots, m_{1}, m_{2}, \ldots ; r_{1}, r_{2}, \ldots, s_{1}, s_{2}, \ldots\right) l^{2\left(h_{k_{1}}+\bar{h}_{k_{1}}\right)}, \\
h_{k_{1}} & =h_{l_{1}}+\sum n, \quad h_{k_{2}}=h_{l_{2}}+\sum m
\end{aligned}
$$

where $A(\ldots)$ is a numerical coefficient.

Open Access. This article is distributed under the terms of the Creative Commons Attribution License (CC-BY 4.0), which permits any use, distribution and reproduction in any medium, provided the original author(s) and source are credited.

\footnotetext{
${ }^{23}$ Here $\Lambda(z)=: T T:(z)-\frac{3}{10} \partial_{z}^{2} T$ is the level 4 quasiprimary descendent of the identity.
} 


\section{References}

[1] A. Polkovnikov, K. Sengupta, A. Silva and M. Vengalattore, Nonequilibrium dynamics of closed interacting quantum systems, Rev. Mod. Phys. 83 (2011) 863 [arXiv:1007.5331] [INSPIRE].

[2] V. Balasubramanian et al., Thermalization of strongly coupled field theories, Phys. Rev. Lett. 106 (2011) 191601 [arXiv:1012.4753] [INSPIRE].

[3] H. Liu and S.J. Suh, Entanglement growth during thermalization in holographic systems, Phys. Rev. D 89 (2014) 066012 [arXiv: 1311.1200] [INSPIRE].

[4] S. Bhattacharyya and S. Minwalla, Weak field black hole formation in asymptotically AdS spacetimes, JHEP 09 (2009) 034 [arXiv:0904.0464] [INSPIRE].

[5] E. Caceres, A. Kundu, J.F. Pedraza and D.-L. Yang, Weak field collapse in AdS: introducing a charge density, JHEP 06 (2015) 111 [arXiv:1411.1744] [INSPIRE].

[6] P. Bizon and A. Rostworowski, On weakly turbulent instability of Anti-de Sitter space, Phys. Rev. Lett. 107 (2011) 031102 [arXiv:1104.3702] [INSPIRE].

[7] O.J.C. Dias, G.T. Horowitz and J.E. Santos, Gravitational turbulent instability of Anti-de Sitter space, Class. Quant. Grav. 29 (2012) 194002 [arXiv:1109.1825] [INSPIRE].

[8] V. Balasubramanian, A. Buchel, S.R. Green, L. Lehner and S.L. Liebling, Holographic thermalization, stability of Anti-de Sitter space and the Fermi-Pasta-Ulam paradox, Phys. Rev. Lett. 113 (2014) 071601 [arXiv:1403.6471] [INSPIRE].

[9] P. Basu, C. Krishnan and A. Saurabh, A stochasticity threshold in holography and and the instability of $A d S$, arXiv: 1408.0624 [INSPIRE].

[10] B. Craps, O. Evnin and J. Vanhoof, Renormalization group, secular term resummation and AdS (in)stability, JHEP 10 (2014) 048 [arXiv: 1407.6273] [INSPIRE].

[11] B. Craps, O. Evnin and J. Vanhoof, Renormalization, averaging, conservation laws and AdS (in) stability, JHEP 01 (2015) 108 [arXiv: 1412.3249] [INSPIRE].

[12] P. Calabrese and J.L. Cardy, Evolution of entanglement entropy in one-dimensional systems, J. Stat. Mech. (2005) P04010 [cond-mat/0503393] [InSPIRE].

[13] S.R. Das, D.A. Galante and R.C. Myers, Universal scaling in fast quantum quenches in conformal field theories, Phys. Rev. Lett. 112 (2014) 171601 [arXiv:1401.0560] [INSPIRE].

[14] S.R. Das, D.A. Galante and R.C. Myers, Universality in fast quantum quenches, JHEP 02 (2015) 167 [arXiv:1411.7710] [INSPIRE].

[15] T. Barthel and U. Schollwöck, Dephasing and the steady state in quantum many-particle systems, Phys. Rev. Lett. 100 (2008) 100601 [arXiv:0711.4896].

[16] M. Cramer, C.M. Dawson, J. Eisert and T.J. Osborne, Exact relaxation in a class of nonequilibrium quantum lattice systems, Phys. Rev. Lett. 100 (2008) 030602 [cond-mat/0703314].

[17] M. Rigol, V. Dunjko, V. Yurovsky and M. Olshanii, Relaxation in a completely integrable many-body quantum system: an ab Initio study of the dynamics of the highly excited states of $1 d$ lattice hard-core bosons, Phys. Rev. Lett. 98 (2007) 050405 [cond-mat/0604476].

[18] M. Rigol, V. Dunjko and M. Olshanii, Thermalization and its mechanism for generic isolated quantum systems, Nature 452 (2008) 854 [arXiv:0708.1324]. 
[19] A. Iucci and M.A. Cazalilla, Quantum quench dynamics of the Luttinger model, Physical 80 (2009) 063619 [arXiv: 1003.5170].

[20] D. Fioretto and G. Mussardo, Quantum quenches in integrable field theories, New J. Phys. 12 (2010) 055015 [arXiv:0911.3345] [inSPIRE].

[21] P. Calabrese, F.H.L. Essler and M. Fagotti, Quantum quench in the transverse-field Ising chain, Phys. Rev. Lett. 106 (2011) 227203 [arXiv:1104.0154].

[22] P. Calabrese, F.H.L. Essler and M. Fagotti, Quantum quench in the transverse field Ising chain: I. Time evolution of order parameter correlators, J. Stat. Mech. (2012) P07016 [arXiv: 1204.3911].

[23] P. Calabrese, F.H.L. Essler and M. Fagotti, Quantum quenches in the transverse field Ising chain: II. Stationary state properties, J. Stat. Mech. (2012) P07022 [arXiv:1205.2211].

[24] G. Mandal and T. Morita, Quantum quench in matrix models: dynamical phase transitions, selective equilibration and the generalized Gibbs ensemble, JHEP 10 (2013) 197 [arXiv: 1302.0859] [INSPIRE].

[25] B. Bertini, D. Schuricht and F.H.L. Essler, Quantum quench in the sine-Gordon model, J. Stat. Mech. (2014) P10035 [arXiv:1405.4813] [INSPIRE].

[26] M.R. Gaberdiel, K. Jin and E. Perlmutter, Probing higher spin black holes from CFT, JHEP 10 (2013) 045 [arXiv: 1307.2221] [INSPIRE].

[27] M. Beccaria and G. Macorini, Resummation of scalar correlator in higher spin black hole background, JHEP 02 (2014) 071 [arXiv: 1311.5450] [INSPIRE].

[28] S. Datta, J.R. David, M. Ferlaino and S.P. Kumar, Universal correction to higher spin entanglement entropy, Phys. Rev. D 90 (2014) 041903 [arXiv: 1405.0015] [INSPIRE].

[29] P. Calabrese and J. Cardy, Quantum quenches in extended systems, J. Stat. Mech. (2007) P06008 [arXiv:0704.1880] [INSPIRE].

[30] R.K. Gupta and A. Mukhopadhyay, On the universal hydrodynamics of strongly coupled CFTs with gravity duals, JHEP 03 (2009) 067 [arXiv:0810.4851] [INSPIRE].

[31] G. Mandal and N. Sorokhaibam, in preparation.

[32] J. Cardy, Thermalization and revivals after a quantum quench in conformal field theory, Phys. Rev. Lett. 112 (2014) 220401 [arXiv: 1403.3040] [INSPIRE].

[33] P. Calabrese and J.L. Cardy, Time-dependence of correlation functions following a quantum quench, Phys. Rev. Lett. 96 (2006) 136801 [cond-mat/0601225] [INSPIRE].

[34] J.M. Maldacena, Eternal black holes in Anti-de Sitter, JHEP 04 (2003) 021 [hep-th/0106112] [INSPIRE].

[35] T. Hartman and J. Maldacena, Time evolution of entanglement entropy from black hole interiors, JHEP 05 (2013) 014 [arXiv: 1303.1080] [INSPIRE].

[36] P. Caputa, G. Mandal and R. Sinha, Dynamical entanglement entropy with angular momentum and $\mathrm{U}(1)$ charge, JHEP 11 (2013) 052 [arXiv: 1306.4974] [INSPIRE].

[37] M.R. Gaberdiel and R. Gopakumar, An $A d S_{3}$ dual for minimal model CFTs, Phys. Rev. D 83 (2011) 066007 [arXiv:1011.2986] [INSPIRE].

[38] M. Gutperle and P. Kraus, Higher spin black holes, JHEP 05 (2011) 022 [arXiv:1103.4304] [INSPIRE]. 
[39] P. Kraus and E. Perlmutter, Partition functions of higher spin black holes and their CFT duals, JHEP 11 (2011) 061 [arXiv:1108.2567] [INSPIRE].

[40] G. Mandal and S. Thakur, in preparation.

[41] P. Di Francesco, P. Mathieu and D. Senechal, Conformal field theory, Springer, Germany (1997).

[42] J.L. Cardy, Conformal invariance and surface critical behavior, Nucl. Phys. B 240 (1984) 51.

[43] C. Pope, Lectures on $W$ algebras and $W$ gravity, hep-th/9112076.

[44] P. Bouwknegt and K. Schoutens, W symmetry in conformal field theory, Phys. Rept. 223 (1993) 183 [hep-th/9210010] [INSPIRE].

[45] G. Festuccia and H. Liu, The arrow of time, black holes and quantum mixing of large- $N$ Yang-Mills theories, JHEP 12 (2007) 027 [hep-th/0611098] [INSPIRE].

[46] A. Cabo-Bizet, E. Gava, V.I. Giraldo-Rivera and K.S. Narain, Black holes in the $3 D$ higher spin theory and their quasi normal modes, JHEP 11 (2014) 013 [arXiv:1407.5203] [INSPIRE].

[47] D. Birmingham, I. Sachs and S.N. Solodukhin, Conformal field theory interpretation of black hole quasinormal modes, Phys. Rev. Lett. 88 (2002) 151301 [hep-th/0112055] [INSPIRE].

[48] S. Datta and J.R. David, Higher spin quasinormal modes and one-loop determinants in the BTZ black hole, JHEP 03 (2012) 079 [arXiv:1112.4619] [INSPIRE].

[49] G. Mandal, R. Sinha and N. Sorokhaibam, The inside outs of $A d S_{3} / C F T_{2}$ : exact AdS wormholes with entangled CFT duals, JHEP 01 (2015) 036 [arXiv:1405.6695] [INSPIRE].

[50] P. Calabrese and J. Cardy, Entanglement and correlation functions following a local quench: a conformal field theory approach, J. Stat. Mech. (10) P10004 [arXiv:0708.3750].

[51] M. Nozaki, T. Numasawa and T. Takayanagi, Holographic local quenches and entanglement density, JHEP 05 (2013) 080 [arXiv: 1302.5703] [INSPIRE].

[52] G. Mandal, R. Sinha and T. Ugajin, Quantum quench with defects and its holographic dual, in preparation.

[53] P. Calabrese and J.L. Cardy, Entanglement entropy and quantum field theory, J. Stat. Mech. (2004) P06002 [hep-th/0405152] [INSPIRE].

[54] P. Calabrese, J. Cardy and E. Tonni, Entanglement entropy of two disjoint intervals in conformal field theory II, J. Stat. Mech. (2011) P01021 [arXiv: 1011.5482] [INSPIRE]. 